\title{
Vanishing and identities of conformal blocks divisors
}

\author{
Prakash Belkale, Angela Gibney and Swarnava Mukhopadhyay
}

\begin{abstract}
Conformal block divisors in type $\mathrm{A}$ on $\overline{\mathrm{M}}_{0, \mathrm{n}}$ are shown to satisfy new symmetries when levels and ranks are interchanged in non-standard ways. A connection with the quantum cohomology of Grassmannians reveals that these divisors vanish above the critical level.
\end{abstract}

\section{Introduction}

Given a simple Lie algebra $\mathfrak{g}$ and suitable choice of weights $\vec{\lambda}$ for $\mathfrak{g}$ at level $\ell$, there is a globally generated vector bundle $\mathbb{V}_{\mathfrak{g}, \vec{\lambda}, \ell}$ of conformal blocks on the moduli space $\overline{\mathrm{M}}_{0, \mathrm{n}}$ of stable, n-pointed rational curves. Global generation on $\overline{\mathrm{M}}_{0, \mathrm{n}}$ comes from the surjection

$$
\mathbb{A}_{\mathfrak{g}, \vec{\lambda}}=\mathrm{A}_{\mathfrak{g}, \vec{\lambda}} \times \overline{\mathrm{M}}_{0, \mathrm{n}} \rightarrow \mathbb{V}_{\mathfrak{g}, \vec{\lambda}, \ell}, \quad \text { where } \mathrm{A}_{\mathfrak{g}, \vec{\lambda}}=\left(\otimes_{i=1}^{n} V_{\lambda_{i}}\right)_{\mathfrak{g}}=\frac{\otimes_{i=1}^{n} V_{\lambda_{i}}}{\mathfrak{g}\left(\otimes_{i=1}^{n} V_{\lambda_{i}}\right)}
$$

is the vector space of coinvariants, the largest quotient space on which $\mathfrak{g}$ acts trivially. Here $V_{\lambda}$ is the irreducible finite-dimensional representation of $\mathfrak{g}$ with highest weight $\lambda$. Consequently, one obtains a morphism $f_{\mathbb{V}}$ to a Grassmannian variety of $\operatorname{rk} \mathbb{V}_{\mathfrak{g}, \vec{\lambda}, \ell}$ quotients of $\mathrm{A}_{\mathfrak{g}, \vec{\lambda}}$ :

$$
\overline{\mathrm{M}}_{0, \mathrm{n}} \stackrel{f_{\mathbb{V}}}{\longrightarrow} \operatorname{Grass}^{q u o}\left(\mathrm{rk} \mathbb{V}_{\mathfrak{g}, \vec{\lambda}, \ell}, \mathrm{A}_{\mathfrak{g}, \vec{\lambda}}\right) \stackrel{p}{\hookrightarrow} \mathbb{P}^{N-1}, \quad \text { where } N=\left(\begin{array}{c}
\operatorname{rk} \mathbb{A}_{\mathfrak{g}, \vec{\lambda}} \\
\operatorname{rk} \mathbb{V}_{\mathfrak{g}, \vec{\lambda}}
\end{array}\right) .
$$

The composition of $f_{\mathbb{V}}$ with the Plücker embedding $p$ is given by $c_{1}\left(\mathbb{V}_{\mathfrak{g}, \vec{\lambda}, \ell}\right)$. These so-called conformal blocks divisors $\mathbb{D}_{\mathfrak{g}, \vec{\lambda}, \ell}=c_{1}\left(\mathbb{V}_{\mathfrak{g}, \vec{\lambda}, \ell}\right)$ generate a full-dimensional sub-cone in the nef cone.

It is well known that given $\mathfrak{g}$ and $\vec{\lambda}$, there is an integer $\ell_{0}(\mathfrak{g}, \vec{\lambda})$ for which $\mathbb{V}_{\mathfrak{g}, \vec{\lambda}, \ell}$ and $\mathbb{A}_{\mathfrak{g}, \vec{\lambda}}$ are isomorphic for all $\ell>\ell_{0}$. In particular, $\operatorname{rk} \mathbb{A}_{\mathfrak{g}, \vec{\lambda}}=\mathrm{rk} \mathbb{V}_{\mathfrak{g}, \vec{\lambda}, \ell}$, and so the conformal blocks divisor $\mathbb{D}_{\mathfrak{g}, \vec{\lambda}, \ell}$ vanishes and its map contracts everything, for all $\ell>\ell_{0}$.

Here we study two different bounds for $\ell_{0}$ : the critical level $c\left(\mathfrak{s l}_{r+1}, \vec{\lambda}\right)$ and the theta level $\theta(\mathfrak{g}, \vec{\lambda})$. The critical level, which we introduce, is related to an interpretation of the ranks of the bundles with $\mathfrak{g}=\mathfrak{s l}_{r+1}$ in terms of the quantum cohomology of the Grassmannian. If $\ell$ is greater than either bound, then $\mathbb{D}_{\mathfrak{g}, \vec{\lambda}, \ell}=0$ (Theorem 1.3 and Remark 1.5). While equal for $r=1$, the critical and theta levels are generally distinct, reflecting different aspects of the weights $\vec{\lambda}$.

As an application of vanishing, we give sufficient conditions for divisors $\mathbb{D}_{\mathfrak{g}, \vec{\lambda}, \ell}$ to be extremal in the nef cone (Propositions 5.3 and 5.4), and show that the morphisms they define factor

Received 3 April 2014, accepted in final form 14 September 2014.

2010 Mathematics Subject Classification 14H10, 14H60, 14N35, 14D20, 14C20 (primary), $14 \mathrm{E} 30$ (secondary).

Keywords: conformal blocks, generalized theta functions, moduli of curves, quantum cohomology, strange duality. This journal is (C) Foundation Compositio Mathematica 2015. This article is distributed with Open Access under the terms of the Creative Commons Attribution Non-Commercial License, which permits non-commercial reuse, distribution, and reproduction in any medium, provided that the original work is properly cited. For commercial re-use, please contact the Foundation Compositio Mathematica.

P.B. and S.M. were supported on NSF grant DMS-0901249, and A.G. on NSF grant DMS-1201268. 


\section{VANISHING AND IDENTITIES OF CONFORMAL BLOCKS DIVISORS}

through birational contraction maps to Hassett spaces (Theorems 6.2 and 6.3). We find a surprising relationship between pairs of critical level bundles $\mathbb{V}_{\mathfrak{s l}_{r+1}, \vec{\lambda}, \ell}$ and $\mathbb{V}_{\mathfrak{s l}_{\ell+1}, \vec{\lambda}^{T}, r}$, where $\ell$ is the critical level for $\left(\mathfrak{s l}_{r+1}, \vec{\lambda}\right)$. In this case, $r$ is the critical level for the pair $\left(\mathfrak{s l}_{\ell+1}, \vec{\lambda}^{T}\right)$, where $\vec{\lambda}^{T}=\left(\lambda_{1}^{T}, \ldots, \lambda_{n}^{T}\right)$ and the $\lambda_{i}^{T}$ are the representations given by the transposed Young diagrams associated with the $\lambda_{i}$ as described in Section 1.1.1. Namely, while the bundles $\mathbb{V}_{\mathfrak{s l}_{r+1}, \vec{\lambda}, \ell}$ and $\mathbb{V}_{\mathfrak{s l}_{\ell+1}, \vec{\lambda}^{T}, r}$ are not isomorphic, their first Chern classes are equal, and the images of the maps they define are related by Grassmann duality (Proposition 1.6).

In addition to the identity $\mathbb{D}_{\mathfrak{s l}_{r+1}, \vec{\lambda}, \ell}=\mathbb{D}_{\mathfrak{s l}_{\ell+1}, \vec{\lambda}^{T}, r}$ for critical level pairs, we show that $\mathbb{D}_{\mathfrak{s l}_{r+1}, \vec{\lambda}, \ell}=\mathbb{D}_{\mathfrak{s l}_{r+1}, \vec{\lambda}^{*}, \ell}$ (Proposition 13.1). Here $\vec{\lambda}^{*}=\left(\lambda_{1}^{*}, \ldots, \lambda_{n}^{*}\right)$ and $\lambda^{*}$ denotes the highest weight of $\left(V_{\lambda}\right)^{*}$. Taken together, these identities enhance our criteria for detecting the vanishing of the conformal block divisors, and hence improve our understanding of the nef cone.

\subsection{Definitions and precise statement of results}

We now state our results, outline the paper and our methods, and put the work into context.

1.1.1 Notation. For a finite-dimensional simple Lie algebra $\mathfrak{g}$ and a positive integer $\ell$ (called the level), denote by $P_{\ell}(\mathfrak{g})$ the set of dominant integral weights $\lambda$ with $(\lambda, \theta) \leqslant \ell$. Here $\theta$ is the highest root and ( , ) is the Killing form, normalized so that $(\theta, \theta)=2$. If $H_{\theta}$ is the co-root corresponding to the highest root $\theta$, then $(\lambda, \theta)=\lambda\left(H_{\theta}\right)$.

To a triple $(\mathfrak{g}, \vec{\lambda}, \ell)$ such that $\vec{\lambda} \in P_{\ell}(\mathfrak{g})^{n}$, there corresponds a vector bundle of conformal blocks $\mathbb{V}_{\mathfrak{g}, \vec{\lambda}, \ell}$ on the moduli stack $\overline{\mathcal{M}}_{g, n}$ [TUY89] (see also [Tsu93, Sor96, Fak12]).

Finite-dimensional irreducible polynomial representations for $\mathrm{GL}_{r+1}$ are parameterized by Young diagrams $\lambda=\left(\lambda^{(1)} \geqslant \lambda^{(2)} \geqslant \cdots \geqslant \lambda^{(r)} \geqslant \lambda^{(r+1)} \geqslant 0\right)$. Young diagrams $\lambda$ and $\mu$ give the same representation of $\mathrm{SL}_{r+1}$ (or, equivalently, $\mathfrak{s l}_{r+1}$ ) if $\lambda^{(a)}-\mu^{(a)}$ is a constant independent of $a$. We use the notation $|\lambda|=\sum_{i=1}^{r} \lambda^{(i)}$. We say that $\lambda \in P_{\ell}\left(\mathfrak{s l}_{r+1}\right)$ if and only if $\lambda^{(1)}-\lambda^{(r+1)} \leqslant \ell$. We refer to $\lambda$ as normalized if $\lambda^{(r+1)}=0$. The normalization of $\lambda$ is $\lambda-\lambda^{(r+1)} \cdot(1,1, \ldots, 1)$. Also note that $(\lambda, \theta)=\lambda\left(H_{\theta}\right)=\lambda^{(1)}-\lambda^{(r+1)}$.

\subsubsection{The critical level}

Definition 1.1. Let $\vec{\lambda}=\left(\lambda_{1}, \ldots, \lambda_{n}\right)$ be an $n$-tuple of normalized integral weights for $\mathfrak{s l}_{r+1}$, assume that $r+1$ divides $\sum_{i=1}^{n}\left|\lambda_{i}\right|$, and define the critical level for the pair $\left(\mathfrak{s l}_{r+1}, \vec{\lambda}\right)$ to be

$$
c\left(\mathfrak{s l}_{r+1}, \vec{\lambda}\right)=-1+\frac{1}{r+1} \sum_{i=1}^{n}\left|\lambda_{i}\right| .
$$

One can define $c\left(\mathfrak{s l}_{r+1}, \vec{\lambda}\right)$ in general, by replacing each $\lambda_{i}$ with its normalization.

Remark 1.2. (i) The non-zeroness of $\operatorname{rk} \mathbb{A}_{\mathfrak{g}, \vec{\lambda}}\left(\right.$ similarly, of $\operatorname{rk} \mathbb{V}_{\mathfrak{g}, \vec{\lambda}, \ell}$ ) for $\mathfrak{g}=\mathfrak{s l}_{r+1}$ is controlled by a non-trivial system of inequalities [Kly98, KT99, Ful00, Bel08a, Bel10]. It is therefore unreasonable to look for an optimal critical level valid for all data.

(ii) The ranks of $\mathbb{A}_{\mathfrak{g}, \vec{\lambda}}$ and $\mathbb{V}_{\mathfrak{g}, \vec{\lambda}, \ell}$ coincide with the ranks of global sections of line bundles over suitable moduli spaces - the moduli spaces of (semistable) filtered vector spaces, and of semistable parabolic bundles, respectively. One may look for levels at which parabolic semistable bundles are necessarily trivial (as bundles), so that these moduli spaces and line bundles are the same, and hence the ranks coincide. The resulting bounds for vanishing are 


\section{Belkale, Gibney and Mukhopadhyay}

weaker than the critical level bounds (by one); see Section 4.1 and Question 4.2.

Proposition 1.3. Suppose $\vec{\lambda} \in P_{\ell}\left(\mathfrak{s l}_{r+1}\right)^{n}$ and $\ell>c\left(\mathfrak{s l}_{r+1}, \vec{\lambda}\right)$. Then $\mathbb{A}_{\mathfrak{s l}_{r+1}, \vec{\lambda}}=\mathbb{V}_{\mathfrak{s l}_{r+1}, \vec{\lambda}, \ell}$ and, in particular, one has $\mathbb{D}_{\mathfrak{s l}_{r+1}, \vec{\lambda}, \ell}=c_{1}\left(\mathbb{V}_{\mathfrak{s l}_{r+1}, \vec{\lambda}, \ell}\right)=0$.

The proof of Proposition 1.3, given in Section 4, follows from an enumerative interpretation of conformal blocks [Wit95] (also S. Agnihotri, Ph.D. thesis, Oxford, 1995) and of classical invariants for $\mathfrak{s l}_{r+1}$ described in Section 3. As is explained in Section 3.2 one can compute the rank of $\mathbb{V}_{\mathfrak{s l}_{r+1}, \vec{\lambda}, \ell}$ by calculating a particular (quantum) product of Schubert cycles in a Grassmannian. In case $\ell>c\left(\mathfrak{s l}_{r+1}, \vec{\lambda}\right)$, the corresponding quantum cohomology structure coefficient equals the classical structure constant, which gives the rank of the bundle of coinvariants.

The critical level is defined only for Lie algebras of form $\mathfrak{g}=\mathfrak{s l}_{r+1}$. The theta level which we define next is valid for arbitrary simple Lie algebras.

1.1.3 The theta level and comparison to the critical level. The theta level, defined below, arises from a description of $\mathbb{V}_{\mathfrak{g}, \vec{\lambda}, \ell}$ as an explicit quotient of $\mathbb{A}_{\mathfrak{g}, \vec{\lambda}}$ [GW86, FSV94, FSV95, Bea96]. For $\mathfrak{g}=\mathfrak{s l}_{r+1}$, this level can be related to critical levels for related data (see Lemma 13.3). Some basic properties of the theta level are explored in [BGM13].

Definition 1.4. Given a pair $(\mathfrak{g}, \vec{\lambda})$, we refer to

$$
\theta(\mathfrak{g}, \vec{\lambda})=-1+\frac{1}{2} \sum\left(\lambda_{i}, \theta\right) \in \frac{1}{2} \mathbb{Z}
$$

as the theta level.

Remark 1.5. (i) It is an immediate consequence of the explicit quotient description of $\mathbb{V}_{\mathfrak{g}, \vec{\lambda}, \ell}$ that if $\ell>\theta(\mathfrak{g}, \vec{\lambda})$ and $\vec{\lambda} \in P_{\ell}(\mathfrak{g})$, then $\mathbb{V}_{\mathfrak{g}, \vec{\lambda}, \ell}=\mathbb{A}_{\mathfrak{g}, \vec{\lambda}}$ and $\mathbb{D}_{\mathfrak{g}, \vec{\lambda}, \ell}=c_{1}\left(\mathbb{V}_{\mathfrak{g}, \vec{\lambda}, \ell}\right)=0$ (see [BGM13] and Lemma 13.3).

(ii) It is easy to see that the theta and critical levels coincide for $\mathfrak{s l}_{2}$. Lemma 13.3(a) shows that the theta level for $\left(\mathfrak{s l}_{r+1}, \vec{\lambda}\right)$ is the average of the critical levels for $\left(\mathfrak{s l}_{r+1}, \vec{\lambda}\right)$ and $\left(\mathfrak{s l}_{r+1}, \vec{\lambda}^{*}\right)$.

1.1.4 Applications of vanishing. A divisor on a projective variety $X$ is nef if it non-negatively intersects all curves on $X$. The set of nef divisors forms the nef cone, denoted by $\operatorname{Nef}(X)$. Properties of $\operatorname{Nef}(X)$ reflect aspects of the morphisms admitted by $X$. The feasibility of studying the birational geometry of a particular space $X$ by way of its nef cone is highly variable. On one extreme, if $X$ is a so-called Mori Dream Space, then $\operatorname{Nef}(X)$ has a finitely number of extremal rays, and each extremal ray is spanned by a base-point-free divisor.

It was recently shown that $\overline{\mathrm{M}}_{0, \mathrm{n}}$ is not a Mori Dream Space for $n \geqslant 13$ [CT13, GK14]. Whether there are an infinite number of distinct extremal rays of the nef cone and whether those rays are spanned by base-point-free divisors are two wide open questions.

Propositions 5.3 and 5.4 give criteria for detecting when a conformal blocks divisor $\mathbb{D}$ lies on an extremal face of the nef cone given by a so-called F-curve. The extremality criteria allow us, in Theorems 6.2 and 6.3, to prove that the morphisms given by certain conformal blocks divisors $\mathbb{D}_{\mathfrak{g}, \vec{\lambda}, \ell}$ factor through maps to Hassett spaces $\overline{\mathrm{M}}_{0, \mathcal{A}}$, where the weight data $\mathcal{A}$ is determined by $\mathfrak{g}, \ell$, and $\vec{\lambda}$.

1.1.5 Relations between divisors. When finding where in the nef cone the conformal blocks divisors reside, we are interested not only in extremality (that results like Propositions 5.3 and 5.4 


\section{VANISHING AND IDENTITIES OF CONFORMAL BLOCKS DIVISORS}

tell us about), but also in questions of independence. An interesting feature of the set of conformal blocks divisors is that together they generate a dense sub-cone of the nef cone. It is natural to wonder whether they cover the entire nef cone, and whether the cone they span is finitely generated. To answer such questions we would like to know their dependence on one another.

We study two types of relations between conformal blocks divisors given by different sets of data. First, in Proposition 13.1, we point out the natural identity between divisors $c_{1}\left(\mathbb{V}_{\mathfrak{g}, \vec{\lambda}, \ell}\right)=$ $c_{1}\left(\mathbb{V}_{\mathfrak{g}, \vec{\lambda}^{*}, \ell}\right)$ that comes from an involution of the Weyl chamber. Second, in Theorem 1.8, we identify pairs of so-called critical level partner divisors $c_{1}\left(\mathbb{V}_{\mathfrak{s l}_{r+1}, \vec{\lambda}, \ell}\right)=c_{1}\left(\mathbb{V}_{\mathfrak{s l}_{\ell+1}, \vec{\lambda}^{T}, r}\right)$. These divisor class identities multiply our extremality results. Namely, Proposition 5.3 holds for $\mathbb{D}_{\mathfrak{s l}_{r+1}, \vec{\lambda}, \ell}$ and $\mathbb{D}_{\mathfrak{s l}_{r+1}, \vec{\lambda}^{*}, \ell}$ (see Proposition 13.1), and Proposition 5.4 holds for $\mathbb{D}_{\mathfrak{s l}_{r+1}, \vec{\lambda}, \ell}$ and $\mathbb{D}_{\mathfrak{s l}_{\ell+1}, \vec{\lambda}^{T}, r}$.

Next, we discuss the critical level identities, which involve a more robust statement about the bundles themselves. To begin with, critical level bundles come in pairs. If $\lambda_{i} \in P_{\ell}\left(\mathfrak{s l}_{r+1}\right)$, then $\lambda_{i}^{T} \in P_{r}\left(\mathfrak{s l}_{\ell+1}\right)$, where $\lambda_{i}^{T}$ is obtained by taking the transpose of the Young diagram associated with the weight $\lambda_{i}$. Since $\left|\lambda_{i}\right|=\left|\lambda_{i}^{T}\right|$, it follow that $\ell=c\left(\mathfrak{s l}_{r+1}, \vec{\lambda}\right)$ if and only if $r=c\left(\mathfrak{s l}_{\ell+1}, \vec{\lambda}^{T}\right)$.

Proposition 1.6. Suppose $\vec{\lambda} \in P_{\ell}\left(\mathfrak{s l}_{r+1}\right)^{n}$, where $\ell=c\left(\mathfrak{s l}_{r+1}, \vec{\lambda}\right)$. Then

(a) one has

$$
\operatorname{rk} \mathbb{V}_{\mathfrak{s l}_{r+1}, \vec{\lambda}, \ell}+\operatorname{rk} \mathbb{V}_{\mathfrak{s l}_{\ell+1}, \vec{\lambda}^{T}, r}=\operatorname{rk}_{\mathfrak{s l}_{r+1}, \vec{\lambda}}=\operatorname{rk}_{\mathfrak{s l}_{\ell+1}, \vec{\lambda}^{T}}
$$

(b) critical level partner divisors are the same:

$$
\mathbb{D}_{\mathfrak{s l}_{r+1}, \vec{\lambda}, \ell}=\mathbb{D}_{\mathfrak{s l}_{\ell+1}, \vec{\lambda}^{T}, r}
$$

Definition 1.1, Theorem 1.3, and Proposition 1.6 (b) were discovered by Fakhruddin for $r=1$ [Fak12]. In loc. cit., he defines the critical level in Section 4.3 and in Remark 5.3 he compares the divisors

$$
c_{1}\left(\mathbb{V}\left(\mathfrak{s l}_{2},\left(i_{1} \omega_{1}, \ldots, i_{n} \omega_{1}\right), \ell\right)\right) \text { and } c_{1}\left(\mathbb{V}\left(\mathfrak{s l}_{\ell+1},\left(\omega_{i_{1}}, \ldots, \omega_{i_{n}}\right), 1\right)\right)
$$

satisfying $\sum_{j=1}^{n} i_{j}=2(\ell+1)$, pointing out that the formulas he derives in Proposition 5.2 and Equation 4.3 establish that the divisors are the same.

Remark 1.7. Our critical level symmetries are different from, but related to, the strange dualities considered in the literature (for example [Bel08b, MO07, NT92, Oud11]). See Section 14.

Proposition 1.6 should be thought of as a corollary to the following result.

Theorem 1.8. Suppose $\vec{\lambda} \in P_{\ell}\left(\mathfrak{s l}_{r+1}\right)^{n}$, where $\ell=c\left(\mathfrak{s l}_{r+1}, \vec{\lambda}\right)$. Then there is a natural isomorphism

$$
\mathbb{A}_{\mathfrak{s l}_{r+1}, \vec{\lambda}}^{*} / \mathbb{V}_{\mathfrak{s l} \mathfrak{l}_{r+1}, \vec{\lambda}, \ell}^{*} \stackrel{\sim}{\rightarrow} \mathbb{V}_{\mathfrak{s l}_{\ell+1}, \vec{\lambda}^{T}, r}
$$

Remark 1.9. Proposition 1.6(a) and Theorem 1.8 hold even on $\overline{\mathrm{M}}_{0,3}$, where the bundles are just vector spaces, giving new statements about their ranks.

To prove Proposition 1.6 and Theorem 1.8, certain structure constant in the cohomology of a Grassmannian is decomposed into sums of two quantum cohomology structure constants for different Grassmannians, yielding (1) (see Section 9.9). 


\section{Belkale, Gibney and Mukhopadhyay}

\subsection{A note on our methods}

The main results of this paper are proved by using relations of conformal blocks to generalized theta functions, and by arguments used in the geometric strange duality theory of vector bundles [Bel08b, MO07, Oud11] and in the study of quantum cohomology of Grassmannians ([Wit95]; see also [Bel10]). The applications use standard intersection theoretic computations on $\overline{\mathrm{M}}_{0, \mathrm{n}}$ and the factorization formulas of [TUY89]. We recommend the Bourbaki article of Sorger [Sor96] for some of the background on conformal blocks.

In [Fak12], Fakhruddin gives explicit formulas for the classes $c_{1}\left(\mathbb{V}_{\mathfrak{g}, \vec{\lambda}, \ell}\right)$. Explicit formulas for the ranks of $\mathbb{V}_{\mathfrak{s l}_{r+1}, \vec{\lambda}, \ell}$ are also known (the Verlinde formula; see Théorème 4.2.2 in [Sor96]). Therefore it is an interesting question to ask whether Propositions 1.3 and 1.6 can be proved using these explicit formulas. It is not clear, however, how to obtain these results from formulas (see Remark 11.6) because of difficulties with factorization data and ranks.

\section{Schubert varieties and the cohomology of Grassmannians}

\subsection{Notation}

We write $[m]=\{1, \ldots, m\}$ for all positive integers $m$. For an $m$-dimensional vector space $W$, denote by $\mathrm{Fl}(W)$ the space of complete flags of vector subspaces of $W$ :

$$
F_{\bullet}: 0 \subsetneq E_{1} \subsetneq E_{2} \subsetneq \cdots \subsetneq E_{m}=W .
$$

The determinant line $\wedge^{m} W$ is denoted by $\operatorname{det} W$. We fix a collection of $n$ distinct and ordered points $S=\left\{p_{1}, \ldots, p_{n}\right\} \subseteq \mathbb{P}^{1}$, and for a vector bundle $\mathcal{W}$ on $\mathbb{P}^{1}$, define $\operatorname{Fl}_{S}(\mathcal{W})=\prod_{p \in S} \operatorname{Fl}\left(\mathcal{W}_{p}\right)$. If $\mathcal{E} \in \mathrm{Fl}_{S}(\mathcal{W})$, we will assume that it is written in the form $\mathcal{E}=\left(E_{\bullet}^{p} \mid p \in S\right)$.

\subsection{Schubert varieties and their tangent spaces}

Let $E_{\bullet} \in \mathrm{Fl}(W)$ be a complete flag in an $M$-dimensional vector space $W$, where $M=r+1+k$. Here we think of $k$ as related to the level $\ell$. Suppose $\lambda$ is a Young diagram that fits into an $(r+1) \times k$ box. It is useful to associate with $\lambda$ an $(r+1)$-element subset $I$ of $[M]$ by $I=\left\{i_{1}<\cdots<i_{r+1}\right\}$, $i_{a}=k+a-\lambda^{(a)}, i_{0}=0, i_{(r+1)+1}=M$. The open Schubert cell $\Omega_{I}^{o}\left(E_{\bullet}\right)=\Omega_{\lambda}^{o}\left(E_{\bullet}\right)$ is defined to be the smooth subvariety of the Grassmannian $\operatorname{Gr}(r+1, W)$ of $(r+1)$-dimensional vector subspaces of $W$ given by

$$
\Omega_{\lambda}^{o}\left(E_{\bullet}\right)=\left\{V \in \operatorname{Gr}((r+1), W) \mid \operatorname{rk}\left(V \cap E_{j}\right)=a \text { for } i_{a} \leqslant j<i_{a+1}, a \in[r+1]\right\} .
$$

The closure $\Omega_{\lambda}^{o}\left(E_{\bullet}\right)$ is denoted by $\Omega_{\lambda}\left(E_{\bullet}\right)$. This is the set of $V \in \operatorname{Gr}(r+1, W)$ such that $\operatorname{rk}\left(V \cap E_{i_{a}}\right) \geqslant a, a \in[r+1]$. The codimension of $\Omega_{\lambda}\left(E_{\bullet}\right)$ in $\operatorname{Gr}(r+1, W)$ is $|\lambda|$.

Suppose $V \in \Omega_{\lambda}^{o}\left(E_{\bullet}\right)$. Let $Q=W / V$. It is easy to see that $V$ and $Q$ each receive induced flags from $E_{\bullet}$; denote these by $F_{\bullet}$ and $G_{\bullet}$, respectively. Then $T \Omega_{\lambda}^{o}\left(E_{\bullet}\right)_{V} \subseteq T \operatorname{Gr}(r+1, W)_{V}=$ $\operatorname{Hom}(V, Q)$ is

$$
T \Omega_{\lambda}^{o}\left(E_{\bullet}\right)_{V}=\left\{\phi \in \operatorname{Hom}(V, Q) \mid \phi\left(F_{a}\right) \subseteq G_{k-\lambda^{(a)}}, a \in[r+1]\right\}
$$

\subsection{Grassmann duality}

Let $W$ be an $M$-dimensional vector space, where $M=r+1+k$. A natural identification $\operatorname{Gr}(r+1, W)=\operatorname{Gr}\left(k, W^{*}\right)$ takes a subspace $V \subseteq W$ to the kernel of the surjective map $W^{*} \rightarrow$ $V^{*}$. There is, similarly, an identification of the complete flag varieties $\mathrm{Fl}(W)$ and $\mathrm{Fl}\left(W^{*}\right)$. A Schubert variety $\Omega_{\lambda}\left(E_{\bullet}\right) \subseteq \operatorname{Gr}(r+1, W)$ can be identified with $\Omega_{\lambda^{T}}\left(E_{\bullet}^{\prime}\right) \subseteq \operatorname{Gr}\left(k, W^{*}\right)$, where $E_{\bullet}$ 


\section{VANISHING AND IDENTITIES OF CONFORMAL BLOCKS DIVISORS}

and $E_{\bullet}^{\prime}$ correspond under the identification of $\mathrm{Fl}(W)$ and $\mathrm{Fl}\left(W^{*}\right)$. A natural group isomorphism $\mathrm{GL}(W) \rightarrow \mathrm{GL}\left(W^{*}\right)$ acts equivariantly on the identifications above.

Let $\lambda$ be a Young diagram which fits into an $(r+1) \times k$ box. Let $\lambda^{c}$ be the complement of the Young diagram $\lambda$. We consider the line bundles $\mathcal{L}_{\lambda^{c}}$ and $\mathcal{L}_{\lambda}$ on $\operatorname{Fl}(W)$ and $\operatorname{Fl}\left(W^{*}\right)$, respectively, as defined in Section 8.1. The line bundle $\mathcal{L}_{\lambda}$ pulls back to $\mathcal{L}_{\lambda^{c}} \otimes \operatorname{det}\left(W^{*}\right)^{k}$ under the natural map from $\mathrm{Fl}(W)$ to $\mathrm{Fl}\left(W^{*}\right)$, equivariantly under the action of groups $\mathrm{SL}(W) \rightarrow \mathrm{SL}\left(W^{*}\right)$, where we note that the line bundle on $\mathrm{Fl}(W)$ with fiber $\operatorname{det}(W)$ is trivial as an equivariant $\mathrm{SL}(W)$-line bundle.

\subsection{Classical cohomology of the Grassmannian}

Recall that the cohomology $H^{*}(X, \mathbb{Z})$ of a Grassmannian $X=\operatorname{Gr}(r+1, M)=\operatorname{Gr}\left(r+1, \mathbb{C}^{M}\right)$, where $M=r+1+\ell$, is a commutative and associative ring, with an additive basis of cycle classes $\sigma_{\mu}$ of Schubert varieties $\Omega_{\mu}\left(E_{\bullet}\right)$. Here $\mu$ runs through all Young diagrams which fit into an $(r+1) \times \ell$ grid $\left(\mu\right.$ need not be normalized). Note that $\sigma_{\mu} \in H^{2|\mu|}(X, \mathbb{Z})$. The class of a point $[\mathrm{pt}]$ is $\sigma_{(\ell, \ell, \ldots, \ell)}$.

The coefficient of $\sigma_{\lambda_{n}^{c}}\left(\lambda_{n}^{c}\right.$ is the complement of $\lambda_{n}$ in an $(r+1) \times \ell$ box $)$ in the product

$$
\sigma_{\lambda_{1}} \cdot \sigma_{\lambda_{2}} \cdots \sigma_{\lambda_{n-1}} \in H^{*}(X)=H^{*}(X, \mathbb{Z})
$$

has the following enumerative interpretation: Pick a general element $\left(E_{\bullet}^{1}, \ldots, E_{\bullet}^{n}\right)$ of $\mathrm{Fl}(W)^{n}$, where $W=\mathbb{C}^{M}$, and count the number of points in the intersection

$$
\cap_{i=1}^{n} \Omega_{\lambda_{i}}\left(E_{\bullet}^{i}\right) .
$$

One counts this as zero if this number is infinite.

\subsection{Quantum Cohomology of the Grassmannian}

We suggest [Ber97] for the basic notions of quantum cohomology. As an abelian group, the quantum cohomology group of $X=\operatorname{Gr}(r+1, M)=\operatorname{Gr}\left(r+1, \mathbb{C}^{M}\right)$, where $M=(r+1)+k$, is

$$
Q H^{*}(X)=Q H^{*}(X, \mathbb{Z})=H^{*}(X, \mathbb{Z}) \otimes \mathbb{Z}[q] .
$$

The multiplication in this graded commutative and associative ring is $q$-linear $\left(\sigma_{\nu}\right.$ has degree $|\nu|$ and $q$ has degree $M)$. The coefficient of $q^{d} \sigma_{\lambda_{n}^{c}}\left(\lambda_{n}^{c}\right.$ is the complement of $\lambda_{n}$ in an $(r+1) \times k$ box $)$ in the quantum product

$$
\sigma_{\lambda_{1}} \star \sigma_{\lambda_{2}} \star \cdots \star \sigma_{\lambda_{n-1}} \in Q H^{*}(X)
$$

has the following enumerative interpretation: Fix distinct points $p_{1}, \ldots, p_{n} \in \mathbb{P}^{1}$ and a general element $\left(E_{\bullet}^{1}, \ldots, E_{\bullet}^{n}\right)$ of $\operatorname{Fl}(W)^{n}$, where $W=\mathbb{C}^{M}$, and count the number of maps $f: \mathbb{P}^{1} \rightarrow X$ of degree $d$ such that

$$
f\left(p_{i}\right) \in \Omega_{\lambda_{i}}\left(E_{\bullet}^{i}\right) \subseteq X \quad \forall i \in[n],
$$

where if this number is infinite, the count is considered zero. Setting $q=0$ in the product $\sigma_{\mu} \star \sigma_{\nu}$, we recover the classical product $\sigma_{\mu} \cdot \sigma_{\nu} \in H^{*}(X)$.

\section{Witten's theorem}

Definition 3.1. A vector bundle $\mathcal{W}$ on $\mathbb{P}^{1}$ is said to be evenly split if $\mathcal{W}=\oplus_{j=1}^{N} \mathcal{O}_{\mathbb{P}^{1}}\left(a_{j}\right)$ with $\left|a_{i}-a_{j}\right| \leqslant 1$ for $0<i<j \leqslant N$.

Remark 3.2. It is easy to see that there are evenly split bundles of every degree and (non-zero) 


\section{Belkale, Gibney and Mukhopadhyay}

rank. These bundles are generic bundles of the given degree and rank.

Theorem 3.3 Witten's Dictionary. Given $\vec{\lambda} \in P_{\ell}\left(\mathfrak{s l}_{r+1}\right)^{n}$, write $\sum_{i=1}^{n}\left|\lambda_{i}\right|=(r+1)(\ell+s) \in \mathbb{Z}$. Let $\mathcal{W}$ be an evenly split vector bundle of rank $r+1+\ell$ and degree $s$, and let $\mathcal{E} \in \mathrm{Fl}_{S}(\mathcal{W})$ be a general point. Then $\mathrm{rk} \mathbb{V}_{\mathfrak{s l}_{r+1}, \vec{\lambda}, \ell}$ is equal to the cardinality of the set

$$
\left\{\begin{array}{ll}
\text { subbundles } \mathcal{V} \subset \mathcal{W}: \quad & \operatorname{deg}(\mathcal{V})=0, \operatorname{rk}(\mathcal{V})=r+1, \text { and } \\
& \mathcal{V}_{p_{i}} \in \Omega_{\lambda_{i}}\left(E_{\bullet}^{p_{i}}\right) \subseteq \operatorname{Gr}\left(r+1, \mathcal{W}_{p_{i}}\right), i \in[n]
\end{array}\right\},
$$

which is finite by Kleiman transversality (see Section 2.4 of [Bel08a] for the application of Kleiman's theorem [Kle74]).

Remark 3.4. See [Bel08a, Theorem 3.6, Eq (3.10) and Remark 3.8] for a proof of Witten's Dictionary modeled on the proof of its classical counterpart described just after Remark 3.1 below (cf. [Ful00, Section 6.2]).

Remark 3.5. In the original form of Witten's Dictionary one has quantum cohomology structure coefficients (at any degree $d$ ) appear on one side, and the ranks of Verlinde bundles at degree $-d$ appear on the other side (the underlying bundle of parabolic bundles has degree $-d$, which may not be zero). We choose to (cyclically) twist both sides so that the plain degree of the parabolic bundles is now zero and give conformal blocks as in our paper ([Bel08a] explains how to twist the enumerative side so that instead of counting subbundles of the trivial bundle (as in quantum cohomology), one counts subbundles of arbitrary evenly split bundles).

\subsection{Witten's Dictionary, classical analogue}

The classical counterpart of Witten's Dictionary, which goes back at least to L. Lesieur [Les47] (also [Ful00, Section 6.2]) is the following. Let $\lambda_{1}, \ldots, \lambda_{n}$ be Young diagrams that each fit into an $(r+1) \times \ell$ box (not necessarily normalized), and let $V_{\lambda_{i}}$ be the irreducible finite dimensional representation of $\mathfrak{g}$ with highest weight $\lambda_{i}$. Suppose further $\sum_{i=1}^{n}\left|\lambda_{i}\right|=(r+1) \ell$. Then the dimension of the vector space of co-invariants $\left(V_{\lambda_{1}} \otimes \ldots \otimes V_{\lambda_{n}}\right)_{\mathfrak{s l}_{r+1}}$ equals the number of points in

$$
\bigcap_{i=1}^{n} \Omega_{\lambda_{i}}\left(E_{\bullet}^{i}\right) \subseteq \operatorname{Gr}(r+1, W),
$$

where $W$ is a vector space of dimension $(r+1)+\ell$, and $\left(E_{\bullet}^{1}, \ldots, E_{\bullet}^{n}\right)$ is a general element of $\operatorname{Fl}(W)^{n}$. We often refer to this dimension as the rank of the constant bundle $\mathbb{A}_{\mathfrak{s l}_{r+1}, \vec{\lambda}}=$ $\left(V_{\lambda_{1}} \otimes \ldots \otimes V_{\lambda_{n}}\right)_{\mathfrak{s l}_{r+1}} \times \overline{\mathrm{M}}_{0, \mathrm{n}}$ introduced earlier.

We note that the dimension of the vector space of classical co-invariants above also equals the multiplicity of the $\mathfrak{s l}_{r+1}\left(\right.$ or $\left.\mathrm{GL}_{r+1}\right)$ representation $V_{\lambda_{n}^{c}}$ in the tensor product $V_{\lambda_{1}} \otimes \ldots \otimes V_{\lambda_{n-1}}$. Here $\lambda_{n}^{c}$ is the complement of the Young diagram $\lambda_{n}$ in an $(r+1) \times \ell$ grid (and flipped over) and corresponds to the dual of $V_{\lambda_{n}}$ as an $\mathfrak{s l}_{r+1}$ representation. Note that $r+1$ and $\ell$ are fine-tuned to the data of $\lambda_{i}$ by our assumption that $\sum_{i=1}^{n}\left|\lambda_{i}\right|=(r+1) \ell$.

\subsection{Cohomological form of Witten's Dictionary}

To determine the rank of a conformal block $\mathbb{V}_{\mathfrak{s l}_{r+1}, \vec{\lambda}, \ell}$ in terms of quantum cohomology of Grassmannians, write $\sum\left|\lambda_{i}\right|=(r+1)(\ell+s)$. By [Bel08a, Section 3.5], one proceeds as follows.

If $s<0$, then the rank of $\mathbb{V}_{\mathfrak{s l}_{r+1}, \vec{\lambda}, \ell}$ coincides with the rank of the classical coinvariant $\mathbb{A}_{\mathfrak{s l}_{r+1}, \vec{\lambda}}$. 


\section{VANISHING AND IDENTITIES OF CONFORMAL BLOCKS DIVISORS}

If $s \geqslant 0$, let $\lambda=(\ell, 0, \ldots, 0)$. The rank of $\mathbb{V}_{\mathfrak{s l}_{r+1}, \vec{\lambda}, \ell}$ is the coefficient of $q^{s}[\mathrm{pt}]=q^{s} \sigma_{(\ell, \ell, \ldots, \ell)}$ in

$$
\sigma_{\lambda_{1}} \star \cdots \star \sigma_{\lambda_{n}} \star \sigma_{\lambda}^{s} \in Q H^{*}(Y),
$$

where $Y=\operatorname{Gr}(r+1, r+1+\ell)$ and $\sigma_{\lambda}^{s}$ is the $s$-fold quantum $\star$ product of $\sigma_{\lambda}$. We can write the multiplicity above also as the coefficient of $q^{s} \sigma_{\lambda_{n}^{c}}\left(\lambda_{n}^{c}\right.$ is the complement of $\lambda_{n}$ in an $(r+1) \times \ell$ box) in

$$
\sigma_{\lambda_{1}} \star \sigma_{\lambda_{2}} \star \cdots \star \sigma_{\lambda_{n-1}} \star \sigma_{\lambda}^{s} \in Q H^{*}(Y) .
$$

Remark 3.6. The coefficient of $q^{s}[\mathrm{pt}]$ in the quantum product $\sigma_{\lambda_{1}} \star \cdots \star \sigma_{\lambda_{n}}$ is denoted by $\left\langle\sigma_{\lambda_{1}}, \sigma_{\lambda_{2}}, \ldots, \sigma_{\lambda_{n}}\right\rangle_{s}$.

\section{The proof of vanishing above the critical level}

We now prove Proposition 1.3. Let $\tilde{\ell}=\ell\left(\mathfrak{s l}_{r+1}, \vec{\lambda}\right)+1$. We divide the proof into two cases:

(1) The Young diagrams $\lambda_{1}, \ldots, \lambda_{n}$ are in $P_{\tilde{\ell}}\left(\mathfrak{s l}_{r+1}\right)$;

(2) Some $\lambda_{i}$, say $\lambda_{1}$, is not in $P_{\tilde{\ell}}\left(\mathfrak{s l}_{r+1}\right)$.

In case (1), it suffices to prove that $\operatorname{rk} \mathbb{V}_{\mathfrak{s l}_{r+1}, \vec{\lambda}, \tilde{\ell}}=\operatorname{rk} \mathbb{A}_{\mathfrak{s l}_{r+1}, \vec{\lambda}}$. The enumerative translation of $\operatorname{rk} \mathbb{V}_{\mathfrak{s l}_{r+1}, \vec{\lambda}, \tilde{\ell}}$ is the following (see Section 3): Let $\mathcal{W}$ be an evenly split bundle of rank $N=r+\tilde{\ell}+1$ and degree $s=0$; that is, $\mathcal{W}=\mathcal{O}^{\oplus N}=W \otimes \mathcal{O}$ for an $N$-dimensional vector space $W$. Pick a general point $\mathcal{E} \in \mathrm{Fl}_{S}(\mathcal{W})$ (see Section 2.1). The enumerative problem is to count (a finite list by Kleiman transversality) subbundles $\mathcal{V}$ of $\mathcal{W}$ of degree zero and rank $r+1$ such that

$$
\mathcal{V}_{p_{i}} \in \Omega_{\lambda_{i}}\left(E_{\bullet}^{p_{i}}\right) \subseteq \operatorname{Gr}\left(r+1, \mathcal{W}_{p_{i}}\right), \quad i \in[n] .
$$

Any such subbundle is clearly trivial, that is, of the form $V \otimes \mathcal{O}$ for some $r+1$-dimensional subspace $V \subseteq W$, and our count therefore equals the number of points in

$$
\bigcap_{i=1}^{n} \Omega_{\lambda_{i}}\left(E_{\bullet}^{i}\right) \subseteq \operatorname{Gr}(r+1, W),
$$

where $\operatorname{rk} W=N$, and $\left(E_{\bullet}^{1}, \ldots, E_{\bullet}^{n}\right)$ is a general element of $\operatorname{Fl}(W)^{n}$. By Section 3.1, this classical count is $\operatorname{rk} \mathbb{A}_{\mathfrak{s l}_{r+1}, \vec{\lambda}}$ and we are done.

Remark 4.1. We could have argued cohomologically as follows: The rank of $\mathbb{V}\left(\mathfrak{s l}_{r+1}, \vec{\lambda}, \tilde{\ell}\right)$ is, by Section 3.2, the coefficient of [pt] in the quantum product

$$
\sigma_{\lambda_{1}} \star \sigma_{\lambda_{2}} \star \cdots \star \sigma_{\lambda_{n}} \in Q H^{*}(Y),
$$

where $Y=\operatorname{Gr}(r+1, r+1+\tilde{\ell})$, since $s=0$ in our case. This is the classical part of quantum cohomology, and our coefficient equals the rank of $\mathbb{A}_{\mathfrak{s l}_{r+1}, \vec{\lambda}}$, as desired.

In case (2), we claim that $\mathbb{A}_{\mathfrak{s l}_{r+1}, \vec{\lambda}}$ is zero, and hence all $\mathfrak{s l}_{r+1}$-conformal blocks bundles and divisors for $\vec{\lambda}$ are zero. Assume that $k=\lambda_{1}^{(1)}>\tilde{\ell}$ is the maximum of the $\lambda_{i}^{(1)}$ and write $\sum_{i=1}^{n}\left|\lambda_{i}\right|=(r+1) k-p(r+1)$ so that the $\lambda_{i}$ fit into boxes of size $(r+1) \times k$ and $p>0$. Therefore, if we set $\mu_{1}=\cdots=\mu_{p}=(1,1, \ldots, 1)$, then $\sum_{i=1}^{n}\left|\lambda_{i}\right|+\sum_{j=1}^{p}\left|\mu_{j}\right|=(r+1) k$. The representation $V_{(1, \ldots, 1)}$ of $\mathrm{GL}(r+1)$ is trivial as a representation of $\mathfrak{s l}_{r+1}$. Therefore, applying the classical theorem relating intersection numbers for $X=\operatorname{Gr}(r+1, r+1+k)$ and invariants for $\mathfrak{s l}_{r+1}$, we find that the rank of $\mathbb{A}_{\mathfrak{s l}_{r+1}, \vec{\lambda}}$ equals the multiplicity of the class of a point in the cup 
product

$$
\sigma_{\lambda_{1}} \cdots \sigma_{\lambda_{n}} \cdot \sigma_{(1,1, \ldots, 1)}^{p} \in H^{*}(X)
$$

But it is easy to see that the cup product $\sigma_{\lambda_{1}} \cdot \sigma_{(1,1, \ldots, 1)}$ equals zero in $H^{*}(X)$. If $V \in$ $\Omega_{\lambda_{1}}\left(F_{\bullet}\right) \cap \Omega_{(1,1, \ldots, 1)}\left(G_{\bullet}\right)$ with $F_{\bullet}, G_{\bullet}$ in general position, then $V \subseteq G_{r+k}$ and $V \supseteq F_{1}$ (since $\lambda_{1}^{(1)}=k$ ). But $F_{1}$ is not a subset of $G_{r+k}$, therefore no such $V$ exists and hence the cup product is zero.

We could have argued also as follows: According to the classical Pieri rule (see, for example, [Ful00]), $\sigma_{\lambda} \cdot \sigma_{b, 0, \ldots, 0}$ is a sum $\sum \sigma_{\mu}$ over $\mu$ which can obtained from $\lambda$ by addition of $b$ boxes, no two in the same column. Since the first column of $\lambda_{1}^{T}$ is already of the full length $k, \sigma_{\lambda_{1}^{T}} \cdot \sigma_{(r+1,0, \ldots, 0)}=$ $0 \in H^{*}(\operatorname{Gr}(k, r+1+k))$, and the desired vanishing holds by Grassmann duality.

\subsection{Parabolic semistability}

The duals of spaces of conformal blocks $\mathbb{V}_{\mathfrak{s l}_{r+1}, \vec{\lambda}, \ell}$ over points of $\mathrm{M}_{0, n}$ can be identified with global sections of certain line bundles on suitable moduli spaces of semi-stable parabolic bundles on $\mathbb{P}^{1}$. We note that it is not necessary (but perhaps sufficient) for all parabolic semistable bundles to have trivial underlying bundles for $\mathbb{V}_{\mathfrak{s l}_{r+1}, \vec{\lambda}, \ell}$ to be equal to the trivial bundle $\mathbb{A}_{\mathfrak{s l}_{r+1}, \vec{\lambda}}$ (see Remark 11.5 for an example). As has been pointed out to us by an anonymous referee, it is instructive to determine conditions on $\ell$ so that any semistable parabolic bundle $\mathcal{V}$ with weights $\left|\lambda_{i}\right| / \ell$ has the trivial splitting type. Suppose that the underlying bundle $\mathcal{V}$ has $\mathcal{O}(a)$ (with $a>0$ ) as a direct summand, and that $\mathcal{O}(a)$ meets the flags of $\mathcal{V}$ generically. For the subbundle $\mathcal{O}(a)$ to contradict semistability, by an easy calculation, we get $1+\frac{1}{1} \cdot 0>0+\frac{1}{(r+1)} \cdot \sum \frac{\left|\lambda_{i}\right|}{\ell}$, which can happen only when $\ell>\frac{1}{r+1} \sum\left|\lambda_{i}\right|=c\left(\mathfrak{s l}_{r+1}, \vec{\lambda}\right)+1$.

This parabolic method misses $\ell=c\left(\mathfrak{s l}_{r+1}, \vec{\lambda}\right)+1$ : Proposition 1.3 says that $\mathbb{V}_{\mathfrak{s l}_{r+1}, \vec{\lambda}, \ell}$ coincides with coinvariants for $\ell=c\left(\mathfrak{s l}_{r+1}, \vec{\lambda}\right)+1$ whereas this argument is inconclusive. Therefore, the critical level bound is strictly better that the bound obtained via parabolic semistability by one. In fact, for $\ell=c\left(\mathfrak{s l}_{r+1}, \vec{\lambda}\right)+1$ there are semistable parabolic bundles with non-trivial splitting type (see, for example, Example (2) in Table 7, and Remark 11.5). Theorem 1.8 and many others require the critical level as defined, and the connection of the critical level to quantum cohomology is decisive. However, we believe such a parabolic semistability argument should give bounds for all Lie algebras $\mathfrak{g}$, and should be further developed.

We ask the following natural question.

Question 4.2 . Given a triple $(\mathfrak{g}, \vec{\lambda}, \ell)$ with $\vec{\lambda} \in P_{\ell}(\mathfrak{g})^{n}$, what are necessary and sufficient conditions so that $\operatorname{rk} \mathbb{V}_{\mathfrak{g}, \vec{\lambda}, \ell}=\operatorname{rk} \mathbb{A}_{\mathfrak{g}, \vec{\lambda}}$ ?

This answer must be subtle. For example, Corollary 1.6 says that it is sufficient to take $\ell$ greater than the critical level, but that if $\ell$ is equal to the critical level, then in order to have this rank identity it is necessary that the critical level partner bundle have rank zero. A look at the first four lines of Table 7 shows that sometimes this happens, while other times it does not. Another sufficient condition is given by the theta level (Remark 1.5). 


\section{VANISHING AND IDENTITIES OF CONFORMAL BLOCKS DIVISORS}

\section{First application: extremality of the $\mathbb{D}=\mathbb{D}_{\mathfrak{g}, \vec{\lambda}, \ell}$ in the nef cone}

In this section we give criteria (in Propositions 5.3 and 5.4) which come from Corollary 1.6 to detect certain so-called F-curves (see Definition 5.1) that get contracted by the associated conformal blocks maps $\phi_{\mathbb{D}}$. This enables us to show in Section 6 that the morphisms $\phi_{\mathbb{D}}$ factor through certain Hassett contractions.

\subsection{Notation}

Definition 5.1. Fix a partition of $[n]=\{1, \ldots, n\}$ into four non-empty sets $N_{1}, N_{2}, N_{3}, N_{4}=$ $[n] \backslash\left(N_{1} \cup N_{2} \cup N_{3}\right)$, and consider the morphism $\overline{\mathrm{M}}_{0,4} \longrightarrow \overline{\mathrm{M}}_{0, \mathrm{n}}$ given by $\left(C,\left(a_{1}, a_{2}, a_{3}, a_{4}\right)\right) \mapsto$ $\left(X,\left(p_{1}, \ldots, p_{n}\right)\right)$, where $X$ is the nodal curve obtained as follows. If $\left|N_{i}\right| \geqslant 2$, then one glues a copy of $\mathbb{P}^{1}$ to the spine $\left(C,\left(a_{1}, a_{2}, a_{3}, a_{4}\right)\right)$ by attaching a point $\left(\mathbb{P}^{1},\left\{p_{j}: j \in N_{i}\right\} \cup\left\{\alpha_{i}\right\}\right) \in M_{0,\left|N_{i}\right|+1}$ to $a_{i}$ at $\alpha_{i}$. If $\left|N_{i}\right|=1$, one does not glue any curve at the point $a_{i}$, but instead labels $a_{i}$ by $p_{i}$. We refer to any element of the numerical equivalence class of the image of this morphism as the F-Curve $F\left(N_{1}, N_{2}, N_{3}\right)$ or as $F\left(N_{1}, N_{2}, N_{3}, N_{4}\right)$, depending on the context.

Conjecture 5.2 (The F-Conjecture). A divisor $D$ on $\overline{\mathrm{M}}_{0, \mathrm{n}}$ is nef if and only if it non-negatively intersects all F-curves.

\subsection{Contraction results}

Proposition 5.3. Suppose $r \geqslant 1, \ell \geqslant 1$, and let $\vec{\lambda}=\left(\lambda_{1}, \ldots, \lambda_{n}\right)$ be an $n$-tuple in $P_{\ell}\left(\mathfrak{s l}_{r+1}\right)$. Let $N_{1}, N_{2}, N_{3}, N_{4}$ be any partition of $[n]$ into four non-empty subsets. Without loss of generality, we may assume that they are ordered so that, if $\lambda\left(N_{j}\right)=\sum_{i \in N_{j}}\left|\lambda_{i}\right|$, then $\lambda\left(N_{1}\right) \leqslant \cdots \leqslant \lambda\left(N_{4}\right)$. If $\sum_{j \in\{1,2,3\}} \lambda\left(N_{j}\right) \leqslant r+\ell$, then the (possibly constant) morphism $\phi_{\mathbb{D}}$ given by the divisor $\mathbb{D}=\mathbb{D}_{\mathfrak{s l}_{r+1}, \vec{\lambda}, \ell}$ contracts $F\left(N_{1}, N_{2}, N_{3}\right)$ and, in particular, $\mathbb{D}$ is extremal in the nef cone.

Proof. The F-curve $F\left(N_{1}, N_{2}, N_{3}\right)$ is contained in the boundary divisor

$$
\Delta_{N_{1} \cup N_{2} \cup N_{3}} \cong \overline{\mathrm{M}}_{0,\left|N_{1} \cup N_{2} \cup N_{3}\right|+1} \times \overline{\mathrm{M}}_{0,\left|N_{4}\right|+1},
$$

and is actually isomorphic to a curve contained in $\overline{\mathrm{M}}_{0,\left|N_{1} \cup N_{2} \cup N_{3}\right|+1}$ under the attaching map, which attaches the leg with $N_{4}$ marked points to the extra attaching point. Therefore, to show that $\phi_{\mathbb{D}}$ contracts $F\left(N_{1}, N_{2}, N_{3}\right)$, it suffices to show that $\mathbb{D}$ is trivial on $\overline{\mathrm{M}}_{0,\left|N_{1} \cup N_{2} \cup N_{3}\right|+1} \times x$ for a fixed $x \in \overline{\mathrm{M}}_{0,\left|N_{4}\right|+1}$. Let $I=N_{1} \cup N_{2} \cup N_{3}$.

When pulled back to $M=\overline{\mathrm{M}}_{0,\left|N_{1} \cup N_{2} \cup N_{3}\right|+1} \times \overline{\mathrm{M}}_{0,\left|N_{4}\right|+1}$, our bundle $\mathbb{V}_{\mathfrak{s l}_{r+1}, \vec{\lambda}, \ell}$ breaks up (by factorization) into a direct sum

$$
\oplus_{\mu \in P_{\ell}} \mathbb{V}_{\mathfrak{s l}} \mathfrak{l}_{r+1},\left\{\lambda_{i}: i \in I\right\} \cup\{\mu\}, \ell \otimes \mathbb{V}_{\mathfrak{s l}_{r+1},\left\{\lambda_{i}: i \in N_{4}\right\} \cup\left\{\mu^{*}\right\}, \ell}
$$

of tensor products of vector bundles pulled back from the two projections of $M$. It therefore suffices to show that $c_{1}\left(\mathbb{V}_{\mathfrak{s} l_{r+1},\left\{\lambda_{i}: i \in I\right\} \cup\{\mu\}, \ell}\right)=0$ for any $\mu \in P_{\ell}$. But $|\mu| \leqslant \ell r$ and

$$
\sum_{i \in I}\left|\lambda_{i}\right|+|\mu| \leqslant \ell+r+\ell r<(\ell+1)(r+1)
$$

and hence $\ell$ is greater than the critical level for $\left(\mathfrak{s l}_{r+1},\left\{\lambda_{i}: i \in I\right\} \cup\{\mu\}\right)$. We may therefore apply Proposition 1.3 to conclude that the desired $c_{1}$ vanishes.

Using Remark 1.5 in place of Corollary 1.6 gives the following general result for arbitrary $\mathfrak{g}$. Proposition 5.4. Suppose $r \geqslant 1, \ell \geqslant 1$, and let $\vec{\lambda}=\left(\lambda_{1}, \ldots, \lambda_{n}\right)$ be an $n$-tuple in $P_{\ell}(\mathfrak{g})$. Let $N_{1}, N_{2}, N_{3}, N_{4}$ be any partition of $[n]$ into four non-empty subsets. Without loss of generality, 
we may assume that they are ordered so that, if $L\left(N_{j}\right)=\sum_{i \in N_{j}}\left(\lambda_{i}, \theta\right)$, then $L\left(N_{1}\right) \leqslant L\left(N_{2}\right) \leqslant$ $L\left(N_{3}\right) \leqslant L\left(N_{4}\right)$. If $\sum_{j \in\{1,2,3\}} L\left(N_{j}\right) \leqslant \ell+1$, then the (possibly constant) morphism $\phi_{\mathbb{D}}$ given by the divisor $\mathbb{D}=\mathbb{D}_{\mathfrak{g}, \vec{\lambda}, \ell}$ contracts the $\mathrm{F}$-Curve $\mathrm{F}\left(N_{1}, N_{2}, N_{3}\right)$, and, in particular, $\mathbb{D}$ is extremal in the nef cone.

\section{Second application: conformal blocks morphisms and Hassett contractions}

As a step towards understanding the images of the morphisms $\phi_{\mathbb{D}}$ for $\mathbb{D}=c_{1}(\mathbb{V}(\mathfrak{g}, \vec{\lambda}, \ell))$, we show that if the weights $\lambda_{i}$ satisfy prescribed conditions (as given in Theorems 6.2 and 6.3), then $\phi_{\mathbb{D}}$ factors through maps to Hassett's moduli spaces $\overline{\mathrm{M}}_{0, \mathcal{A}}$ of stable weighted pointed rational curves, where the weight data is explicitly determined by the $\mathfrak{g}, \ell$, and $\lambda_{i}$.

\subsection{Background on Hassett spaces}

Consider an $n$-tuple $\mathcal{A}=\left\{a_{1}, \ldots, a_{n}\right\}$, with $a_{i} \in \mathbb{Q}, 0<a_{i} \leqslant 1$, such that $\sum_{i} a_{i}>2$. In [Has03], Hassett introduced moduli spaces $\overline{\mathrm{M}}_{0, \mathcal{A}}$, parameterizing families of stable weighted pointed rational curves $\left(C,\left(p_{1}, \ldots, p_{n}\right)\right)$ such that $(1) C$ is nodal away from its marked points $p_{i} ;(2)$ $\sum_{j \in J} a_{i} \leqslant 1$ if the marked points $\left\{p_{j}: j \in J\right\}$ coincide; and (3) if $C^{\prime}$ is an irreducible component of $C$, then $\sum_{p_{i} \in C^{\prime}} a_{i}$ plus the number of nodes on $C^{\prime}$ is greater than two. These Hassett spaces $\overline{\mathrm{M}}_{0, \mathcal{A}}$ receive birational morphisms $\rho_{\mathcal{A}}$ from $\overline{\mathrm{M}}_{0, \mathrm{n}}$ that are characterized entirely by which F-Curves (see Definition 5.1) they contract.

Definition/Lemma 6.1. For any Hassett space $\overline{\mathrm{M}}_{0, \mathcal{A}}$, with $\mathcal{A}=\left\{a_{1}, \ldots, a_{n}\right\}$, there are birational morphisms $\rho_{\mathcal{A}}: \overline{\mathrm{M}}_{0, \mathrm{n}} \longrightarrow \overline{\mathrm{M}}_{0, \mathcal{A}}$ contracting all F-curves $F\left(N_{1}, N_{2}, N_{3}, N_{4}\right)$ satisfying $\sum_{i \in N_{1} \cup N_{2} \cup N_{3}} a_{i} \leqslant 1$ and no other F-curves, where, without loss of generality, we may assume that the leg $N_{4}$ carries the most weight.

\subsection{Results on Hassett spaces}

The following theorems generalize [Fak12, Proposition 4.7], where $\mathfrak{g}=\mathfrak{s l}_{2}$ was considered.

TheOREM 6.2. Let $\mathbb{D}=\mathbb{D}_{\mathfrak{s l}_{r+1}, \vec{\lambda}, \ell}$ be such that:

(i) $0<\left|\lambda_{i}\right| \leqslant \ell+r$ for all $i \in\{1, \ldots, n\}$;

(ii) $\sum_{i=1}^{n}\left|\lambda_{i}\right|>2(r+\ell)$.

Then the morphism $\phi_{\mathbb{D}}$ factors through $\rho_{\mathcal{A}}: \overline{\mathrm{M}}_{0, \mathrm{n}} \longrightarrow \overline{\mathrm{M}}_{0, \mathcal{A}}$, where $\mathcal{A}=\left\{a_{1}, \ldots, a_{n}\right\}$ with $a_{i}=\left|\lambda_{i}\right| /(r+\ell)$.

Proof. For $\mathcal{A}=\left\{a_{1}, \ldots, a_{n}\right\}$ with $a_{i}=\left|\lambda_{i}\right| /(r+\ell)$ as in the hypothesis, the condition $\left|\lambda_{i}\right| \leqslant \ell+r$ guarantees $a_{i} \leqslant 1$ for all $i$, and $\sum_{i=1}^{n}\left|\lambda_{i}\right|>2(r+\ell)$ guarantees $\sum_{i=1}^{n} a_{i}>2$.

By [Fak12, Lemma 4.6], we need to show that any F-curve $F\left(N_{1}, N_{2}, N_{3}, N_{4}\right)$ contracted by $\rho_{\mathcal{A}}$ is also contracted by $\phi_{\mathbb{D}}$. Suppose that $\rho_{\mathcal{A}}$ contracts the F-curve $F\left(N_{1}, N_{2}, N_{3}, N_{4}\right)$, so that, in particular, by Definition/Lemma 6.1, $\sum_{i \in N_{1} \cup N_{2} \cup N_{3}} a_{i} \leqslant 1$. Then

$$
\sum_{j \in\{1,2,3\}} \lambda\left(N_{j}\right)=(r+\ell) \sum_{j \in\{1,2,3\}} a_{j} \leqslant r+\ell,
$$

and hence by Proposition 5.3, $\phi_{\mathbb{D}}$ contracts F-curve $F\left(N_{1}, N_{2}, N_{3}, N_{4}\right)$.

Using Proposition 5.4 in place of Proposition 5.3, we get the following result. 
Theorem 6.3. Let $\mathbb{D}=\mathbb{D}_{\mathfrak{g}, \vec{\lambda}, \ell}$ be such that $\lambda_{i} \neq 0$ for all $i$ and

$$
\sum_{i=1}^{n} \lambda_{i}\left(H_{\theta}\right)>2(\ell+1) .
$$

Then the morphism $\phi_{\mathbb{D}}$ factors through $\rho_{\mathcal{A}}: \overline{\mathrm{M}}_{0, \mathrm{n}} \longrightarrow \overline{\mathrm{M}}_{0, \mathcal{A}}$, where $\mathcal{A}=\left\{a_{1}, \ldots, a_{n}\right\}$ with $a_{i}=\left(\lambda_{i}, \theta\right) /(\ell+1)$.

Note that if $\mathbb{D}=\mathbb{D}_{\mathfrak{g}, \vec{\lambda}, \ell}$ is non-trivial, then by Remark 1.5, $\sum\left(\lambda_{i}, \theta\right) \geqslant 2(\ell+1)$.

\section{Table}

In these examples, computed using [Swi10], $\ell$ is a critical level for the pair $\left(\mathfrak{s l}_{r+1}, \vec{\lambda}\right)$, and Deg (when $n=4$ ) denotes the degree of $\mathbb{V}_{\mathfrak{s l}_{r+1}, \vec{\lambda}, \ell}\left(\right.$ and of $\mathbb{V}_{\mathfrak{s l}_{\ell+1}, \vec{\lambda}^{T}, r}$ ) over $\overline{\mathrm{M}}_{0,4}=\mathbb{P}^{1}$. In particular, Deg $=0$ means $\mathbb{D}_{\mathfrak{s l}_{r+1}, \vec{\lambda}, \ell}=\mathbb{D}_{\mathfrak{s l}_{\ell+1}, \vec{\lambda}^{T}, r}=0$, even when the rank of $\mathbb{V}_{\mathfrak{s l}_{r+1}, \vec{\lambda}, \ell}\left(\right.$ and of $\left.\mathbb{V}_{\mathfrak{s l}_{\ell+1}, \vec{\lambda}^{T}, r}\right)$ is non-zero. A $*$ in the Deg column indicates more than four marked points.

\begin{tabular}{|l|l|l|l|l|l|l|}
\hline Deg & $(r+1, \ell+1)$ & $n$ & $\vec{\lambda}=\left(\lambda_{1}, \ldots, \lambda_{n}\right)$ & $\begin{array}{l}\text { rank } \\
\mathbb{A}\end{array}$ & $\begin{array}{l}\text { rank } \\
\mathbb{V}_{\mathfrak{s l}_{r+1}, \vec{\lambda}, \ell}\end{array}$ & $\begin{array}{l}\text { rank } \\
\mathbb{V}_{\mathfrak{s l}_{\ell+1}, \vec{\lambda}^{T}, r}\end{array}$ \\
\hline$*$ & $(3,2)$ & 6 & $\left(\omega_{1}, \ldots, \omega_{1}\right)$ & 5 & 1 & 4 \\
\hline 1 & $(3,2)$ & 4 & $\left(\omega_{1}, \omega_{1}, \omega_{2}, \omega_{2}\right)$ & 2 & 1 & 1 \\
\hline 0 & $(4,4)$ & 4 & $\left(\omega_{1},\left(2 \omega_{1}+\omega_{3}\right)^{3}\right)$ & 2 & 1 & 1 \\
\hline$*$ & $(3,6)$ & 5 & $\left(2 \omega_{1}+\omega_{2}, \omega_{2}, 2 \omega_{1}, 2 \omega_{2}, 3 \omega_{2}\right)$ & 7 & 7 & 0 \\
\hline$*$ & $(3,5)$ & 5 & $\left(2 \omega_{1}+\omega_{2}, \omega_{2}, 2 \omega_{1}, 2 \omega_{2}, \omega_{1}+\omega_{2}\right)$ & 9 & 8 & 1 \\
\hline 0 & $(4,4)$ & 4 & $\left(\omega_{2}+\omega_{3}, \omega_{1}, \omega_{1}+2 \omega_{2}, 2 \omega_{1}+\omega_{3}\right)$ & 2 & 1 & 1 \\
\hline 0 & $(4,5)$ & 4 & $\left(\omega_{1}, 2 \omega_{1}+\omega_{2}+\omega_{3},\left(3 \omega_{1}+\omega_{3}\right)^{2}\right)$ & 2 & 1 & 1 \\
\hline 1 & $(4,5)$ & 4 & $\left(\omega_{1}+\omega_{3}, 2 \omega_{1}+2 \omega_{2}, 2 \omega_{1}+2 \omega_{2}, 4 \omega_{1}\right)$ & 4 & 1 & 3 \\
\hline$*$ & $(3,6)$ & 8 & $\left(\left(2 \omega_{1}\right)^{6}, \omega_{2}, 2 \omega_{2}\right)$ & 150 & 136 & 14 \\
\hline
\end{tabular}

\section{Proof of Theorem 1.8: main reductions}

For the proof of Theorem 1.8, in addition to [Wit95], we use the geometric procedure of creating invariants from Schubert calculus [Bel04]. Together with a standard formalism, this leads to a duality isomorphism of classical invariant theory (reviewed in Section 8.1):

$$
A_{\mathfrak{s l}_{r+1}, \vec{\lambda}}^{*} \stackrel{\sim}{\rightarrow} A_{\mathfrak{s l}_{\ell+1}, \vec{\lambda}^{T}} .
$$

The proof of Theorem 1.8 then breaks up into two parts:

(i) We prove equality (1) by working over $\mathrm{M}_{0, n}$ and using the connection between quantum cohomology and conformal blocks, and a degeneration argument for Gromov-Witten invariants (but working over a fixed point of $\mathrm{M}_{0, n}$ ).

(ii) We show that the natural morphism $\mathbb{V}_{\mathfrak{s l}_{r+1}^{*}, \vec{\lambda}, \ell}^{*} \rightarrow \mathbb{V}_{\mathfrak{s l}_{\ell+1}, \vec{\lambda}^{T}, r}$ arising from the isomorphism (5) is the zero map. To do so, we invoke the interpretation [Pau96] of $\mathbb{V}_{\mathfrak{s l} l_{r+1}, \vec{\lambda}, \ell}$ as global sections of a line bundle on a moduli space of parabolic bundles (valid over $\mathrm{M}_{0, n}$, but not over $\overline{\mathrm{M}}_{0, \mathrm{n}}$ ). Again, it suffices to work over $\mathrm{M}_{0, n}$. 


\section{Belkale, Gibney and Mukhopadhyay}

These two steps lead to an isomorphism (2). Let $\mathbb{K}_{r+1, \ell, \vec{\lambda}}$ be the kernel of $\mathbb{A}_{\mathfrak{s l}_{r+1}, \vec{\lambda}} \rightarrow \mathbb{V}_{\mathfrak{s l}_{r+1}, \vec{\lambda}, \ell}$. Consider the exact sequences

$$
\begin{gathered}
0 \rightarrow \mathbb{V}_{\mathfrak{s l}_{r+1}, \vec{\lambda}, \ell}^{*} \rightarrow \mathbb{A}_{\mathfrak{s l}_{r+1}, \vec{\lambda}}^{*} \rightarrow \mathbb{K}_{r+1, \ell, \vec{\lambda}}^{*} \rightarrow 0, \\
0 \rightarrow \mathbb{K}_{\ell+1, r, \vec{\lambda}^{T}} \rightarrow \mathbb{A}_{\mathfrak{s l}_{\ell+1}, \vec{\lambda}^{T}} \rightarrow \mathbb{V}_{\mathfrak{s l}_{\ell+1}, \vec{\lambda}^{T}, r} \rightarrow 0 .
\end{gathered}
$$

Step (2) gives rise to vertical arrows from the first exact sequence above to the second. We therefore find a map (2). By the snake lemma, (2) is a surjective morphism of vector bundles of the same rank, hence an isomorphism.

\subsection{Classical strange duality and the map (5)}

We recall from [Bel04] how Schubert calculus of Grassmannians can be used to produce natural bases in the spaces of invariants and hence produces the duality isomorphism (5) by the (stan-

dard) strange duality formalism. The following is in analogy with the tangent space of a Schubert cell $\Omega_{\lambda}^{o}\left(E_{\bullet}\right)$ in a Grassmannian (see Section 3).

Let $V$ and $Q$ be vector spaces of dimensions $r+1$ and $\ell+1$, respectively. Let $N=r+\ell+2$ (as before). Let $\vec{\lambda}=\left(\lambda_{1}, \ldots, \lambda_{n}\right)$ be an $n$-tuple of Young diagrams that each fit into an $(r+1) \times(\ell+1)$ box. Assume $\sum_{i=1}^{n}\left|\lambda_{i}\right|=(r+1)(\ell+1)$. We next define a divisor $D$ in $\operatorname{Fl}(V)^{n} \times \operatorname{Fl}(Q)^{n}$. A point $\left(F_{\bullet}^{1}, \ldots, F_{\bullet}^{n}, G_{\bullet}^{1}, \ldots, G_{\bullet}^{n}\right)$ is an element of $D$ if and only if there exists a non-zero map $\phi: V \rightarrow Q$ such that for $a \in[r+1]$ and $i \in[n]$,

$$
\phi\left(F_{a}^{i}\right) \subseteq G_{\ell+1-\lambda_{i}^{(a)}}^{i}
$$

We will make this precise by defining $D$ as a determinantal scheme (see Section 11) and will also identify $\mathcal{O}(D)$. We choose to write the answers in a symmetric manner. We set $T=Q^{*}$. Note that $\mathrm{Fl}(Q)$ is canonically identified with $\mathrm{Fl}(T)$. For $a \in[r+1]$, let $\mathcal{L}_{a} \in \operatorname{Pic}(\mathrm{Fl}(V))$ be the pull-back of the ample generator (top exterior power of the dual of the universal subbundle) of $\operatorname{Gr}(a, V)$ by the tautological map

$$
\mathrm{Fl}(V) \rightarrow \operatorname{Gr}(a, V), F_{\bullet} \mapsto F_{a} .
$$

For non-negative integers $\nu_{1}, \ldots, \nu_{r+1}$, define a Young diagram $\lambda=\left(\lambda^{(1)}, \ldots, \lambda^{(r+1)}\right)$ by

$$
\lambda^{(a)}=\nu_{a}+\nu_{a+1}+\cdots+\nu_{r+1}
$$

and a line bundle $\mathcal{L}_{\lambda}=\mathcal{L}_{1}^{\nu_{1}} \otimes \ldots \otimes \mathcal{L}_{r+1}^{\nu_{r+1}}$, whose fiber over $F_{\bullet}$ is denoted by $\mathcal{L}_{\lambda}\left(V, F_{\bullet}\right)=\mathcal{L}_{\lambda}\left(F_{\bullet}\right)$.

Proposition 8.1 (Borel-Weil). The following are isomorphic as representations of GL(V):

$$
H^{0}\left(\operatorname{Fl}(V), \mathcal{L}_{\lambda}\right)=V_{\lambda}^{*} .
$$

8.1.1 For a Young diagram $\lambda$, define a vector bundle $\mathcal{P}_{\lambda}$ on $\operatorname{Fl}(V) \times \operatorname{Fl}(Q)$ with fiber over $\left(F_{\bullet}, G_{\bullet}\right)$ given by

$$
\left\{\phi \in \operatorname{Hom}(V, Q) \mid \phi\left(F_{a}\right) \subseteq G_{\ell+1-\lambda^{(a)}}, a \in[r+1]\right\}
$$

and $\mathcal{T}_{\lambda}=\operatorname{Hom}(V, Q) / \mathcal{P}_{\lambda}$

- The rank of $\mathcal{T}_{\lambda}$ equals $|\lambda|$.

- The fiber of the determinant of $\mathcal{T}_{\lambda}$ at a point $\left(F_{\bullet}, G_{\bullet}\right)$ equals $\mathcal{L}_{\lambda}\left(F_{\bullet}\right) \otimes(\operatorname{det} Q)^{r+1} \otimes \mathcal{L}_{\mu}\left(G_{\bullet}\right)$, where $\mu=\left(\mu^{(1)}, \ldots, \mu^{(\ell+1)}\right)$ is the partition transpose to the partition $\left(\ell+1-\lambda^{(r+1)}, \ldots\right.$, $\left.\ell+1-\lambda^{(1)}\right)$. (See [Bel04], and [BK10], formula 16, where $\mu$ is called the $(\ell+1)$-flip of $\lambda$.) 


\section{VANISHING AND IDENTITIES OF CONFORMAL BLOCKS DIVISORS}

- If $\widetilde{G}_{\bullet} \in \mathrm{Fl}(T)$ corresponds to $G_{\bullet} \in \mathrm{Fl}(Q)$ under the identification $\operatorname{Fl}(T)=\operatorname{Fl}(Q)$, we can write the fiber of the determinant of $\mathcal{T}_{\lambda}$ symmetrically as $\mathcal{L}_{\lambda}\left(F_{\bullet}\right) \otimes \mathcal{L}_{\lambda^{T}}\left(\widetilde{G}_{\bullet}\right)$.

8.1.2 One can view $D$ as the zero locus of the determinant of the following morphism on $\operatorname{Fl}(V)^{n} \times \operatorname{Fl}(Q)^{n}$ of vector bundles of the same rank:

$$
\operatorname{Hom}(V, Q) \rightarrow \bigoplus_{i=1}^{n} \operatorname{pr}_{i}^{*} \mathcal{T}_{\lambda_{i}},
$$

where the $\operatorname{pr}_{i}$ are the projection maps.

Recall that if $\mathcal{V} \rightarrow \mathcal{W}$ is a map between vector bundles of the same rank on a scheme $S$, then one gets a canonical element $s \in H^{0}\left(S, \operatorname{det} \mathcal{W} \otimes(\operatorname{det} \mathcal{V})^{*}\right)$. In this way, we get a line bundle on $\operatorname{Fl}(V)^{n} \times \operatorname{Fl}(Q)^{n}=\operatorname{Fl}(V)^{n} \times \operatorname{Fl}(T)^{n}$, where $T=Q^{*}$, with a canonical section $s$ whose zero locus is $D$.

This line bundle has fiber at $\left(F_{\bullet}^{1}, \ldots, F_{\bullet}^{n}, \widetilde{G}_{\bullet}^{1}, \ldots, \widetilde{G}_{\bullet}^{n}\right) \in \mathrm{Fl}(V)^{n} \times \mathrm{Fl}(T)^{n}$ (see Section 8.1.1), equal to

$$
\otimes_{i=1}^{n}\left(\mathcal{L}_{\lambda_{i}}\left(F_{\bullet}^{i}\right) \otimes \mathcal{L}_{\lambda_{i}^{T}}\left(\widetilde{G}_{\bullet}^{i}\right)\right) \otimes(\operatorname{det} V)^{\ell+1} \otimes(\operatorname{det} T)^{r+1} .
$$

We therefore find an element

$$
s \in H^{0}\left(\mathrm{Fl}(V)^{n}, \otimes_{i=1}^{n} \mathcal{L}_{\lambda_{i}}\right)^{\mathrm{SL}(V)} \otimes H^{0}\left(\mathrm{Fl}(T)^{n}, \otimes_{i=1}^{n} \mathcal{L}_{\lambda_{i}^{T}}\right)^{\mathrm{SL}(T)}=A_{\mathfrak{s l}_{r+1}, \vec{\lambda}}^{*} \otimes A_{\mathfrak{s l}_{\ell+1}, \vec{\lambda}^{T}}^{*}
$$

and a duality map

$$
A_{\mathfrak{s l}_{\ell+1}, \vec{\lambda}^{T}} \rightarrow A_{\mathfrak{s l}_{r+1}, \vec{\lambda}}^{*}
$$

\subsection{Relation to Schubert calculus}

We now explain why the duality map (7) above is an isomorphism. Let $W$ be an $N$-dimensional vector space equipped with $n$ flags $H_{\bullet}^{1}, \ldots, H_{\bullet}^{n}$ in general position, where $N=r+\ell+2$. We fix $V$ and $Q$ as above of dimensions $r+1$ and $\ell+1$, respectively. By Kleiman transversality, the intersection $\cap_{i=1}^{n} \Omega_{\lambda_{i}}\left(H_{\bullet}^{i}\right) \subseteq \operatorname{Gr}(r+1, N)$ is finite, and transverse. Let $V_{1}, \ldots, V_{m}$ be the points in this intersection, and let $Q_{1}, \ldots, Q_{m}$ be the corresponding quotients, $Q_{a}=W / V_{a}$. Each of the vector spaces $V_{1}, \ldots, V_{m}, Q_{1}, \ldots, Q_{m}$ receives $n$ canonical induced flags (from $W$ ), and hence gives $\mathrm{GL}(V)$ and $\mathrm{GL}(Q)$ orbits in $\mathrm{Fl}(V)^{n}$ and $\mathrm{Fl}(Q)^{n}$, respectively (by choosing isomorphisms $V_{a} \rightarrow V$ and $Q_{a} \rightarrow Q$, respectively). We can dualize the $\operatorname{GL}(Q)$ orbits to obtain $\operatorname{GL}(T)$ orbits in $\mathrm{Fl}(T)^{n}$ corresponding to each of $Q_{1}, \ldots, Q_{m}$ (see Section 2.3).

Choose orbit representatives $x_{1}, \ldots, x_{m} \in \mathrm{Fl}(V)^{n}$ and $y_{1}, \ldots, y_{m} \in \mathrm{Fl}(T)^{n}$. From the setup, it is known that $m=\operatorname{rk}\left(\otimes_{i=1}^{n} V_{\lambda_{i}}^{*}\right)^{\operatorname{SL}(V)}=\operatorname{rk}\left(\otimes_{i=1}^{n} V_{\lambda_{i}^{T}}^{*}\right)^{\mathrm{SL}(T)}$, and that $s\left(x_{a}, y_{b}\right) \neq 0$ if and only if $a=b$. Therefore the sections $s\left(\cdot, y_{a}\right)$ (well-defined up to scalars) form a basis for $\left(\otimes_{i=1}^{n} V_{\lambda_{i}}^{*}\right)^{\operatorname{SL}(V)}$ (this was the main result of [Bel04]). Hence by the standard strange duality formalism, the duality map (7) is an isomorphism; also see Lemma 8.3 with quotient stacks $M=\operatorname{Fl}(V)^{n} / \mathrm{SL}(V)$ and $N=\operatorname{Fl}(T)^{n} / \mathrm{SL}(T)$ (and $\mathcal{A}$ and $\mathcal{B}$ descents of $\otimes_{i=1}^{n} \mathcal{L}_{\lambda_{i}}$ and $\otimes_{i=1}^{n} \mathcal{L}_{\lambda_{i}^{T}}$ ).

Remark 8.2. We recall the reason for the vanishing $s\left(x_{a}, y_{b}\right)=0$ for $a \neq b$ : The natural non-zero map $\phi: V_{a} \rightarrow Q_{b}$ (inclusion in $W$ followed by projection to $Q_{b}$ ) satisfies the conditions of (6). It is also easy to see that $s\left(x_{a}, y_{a}\right) \neq 0$ : If $\left(x_{a}, y_{a}\right) \in D$, then any map $\phi$ in the definition of $D$ gives us an element in $\cap_{i=1}^{n} T \Omega_{\lambda_{i}}\left(H_{\bullet}^{i}\right)_{V_{a}}=0$ (by transversality).

The map (2) is defined to be the inverse of (7). By Lemma 8.3, we have explicit control of (2) when we lay out a suitable enumerative problem. 


\section{Belkale, Gibney and Mukhopadhyay}

\subsection{A universal situation}

We analyze the "strange duality" setting of schemes (or stacks) $M, N$ equipped with line bundles $\mathcal{A}$ and $\mathcal{B}$ and a section $s$ of $\mathcal{A} \otimes \mathcal{B}$ in some detail (an instance of this has appeared in Section 8.2). We will use this setting again in Section 11. Fix the following data:

(i) Let $M$ and $N$ be schemes (or quotient stacks $X / G$ ) with line bundles $\mathcal{A}$ and $\mathcal{B}$, respectively. Assume that $H^{0}(M, \mathcal{A})$ and $H^{0}(N, \mathcal{B})$ are both finite-dimensional of the same dimension $m$.

(ii) Suppose that we are given a section $s$ of $\mathcal{A} \otimes \mathcal{B}$ on $M \times N$. This gives rise to a (possibly degenerate) "duality" $s \in H^{0}(M, \mathcal{A}) \otimes H^{0}(N, \mathcal{B})$, or a map $D: H^{0}(M, \mathcal{A})^{*} \rightarrow H^{0}(N, \mathcal{B})$.

Now suppose that we are able to manufacture points $x_{1}, \ldots, x_{m} \in M$ and points $y_{1}, \ldots, y_{m} \in$ $N$ such that $s\left(x_{a}, y_{b}\right)$ is zero if $a \neq b$ and non-zero (as an element of $\mathcal{A}_{x_{a}} \otimes \mathcal{B}_{y_{a}}$ ) if $a=b$. Set $\alpha_{a}=s\left(x, y_{a}\right) \in H^{0}(M, \mathcal{A}) \otimes \mathcal{B}_{y_{a}}$ and $\beta_{a}=s\left(x_{a}, y\right) \in \mathcal{A}_{x_{a}} \otimes H^{0}(N, \mathcal{B})$.

LEMMA 8.3. (i) We have

$$
s=\sum_{a=1}^{m} s\left(x_{a}, y_{a}\right)^{-1} \alpha_{a} \beta_{a} .
$$

(ii) The duality map $D: H^{0}(M, \mathcal{A})^{*} \rightarrow H^{0}(N, \mathcal{B})$ is an isomorphism. It carries the element (welldefined up to scalars) "evaluation at $x_{a}$ " in $H^{0}(M, \mathcal{A})^{*}$ to the section $\beta_{a}$ (up to scalars).

Proof. From the given data, it is clear that the elements of $\left\{\alpha_{a}: a \in[m]\right\}$ and of $\left\{\beta_{a}: a \in[m]\right\}$ are linearly independent (we can ignore the twists by constant lines) and hence form a basis of $H^{0}(M, \mathcal{A})$ and $H^{0}(N, \mathcal{B})$, respectively. Therefore, there is an expression of the form

$$
s=\sum_{a=1}^{m} \sum_{b=1}^{m} \gamma_{a, b} \alpha_{a} \beta_{b}
$$

with $\gamma_{a, b} \in \mathcal{A}_{x_{a}}^{-1} \otimes \mathcal{B}_{y_{b}}^{-1}$. Evaluating this equation at points of the form $\left(x_{a}, y_{b}\right)$, we obtain (8). It is easy to see that (ii) follows from (8).

\section{Proof of Proposition 1.6(a)}

An outline of the proof of Proposition 1.6(a), that is, equality (1), is provided in Section 9.2, after some notation has been introduced. The basic idea is to obtain enumerative interpretations for the ranks of $\mathbb{A}_{\mathfrak{s l}_{r+1}, \vec{\lambda}}, \mathbb{V}_{\mathfrak{s l}_{r+1}, \vec{\lambda}, \ell}$, and $\mathbb{V}_{\mathfrak{s l}_{\ell+1}, \vec{\lambda}^{T}, r}$. The reckoning for $\mathbb{A}_{\mathfrak{s l}_{r+1}, \vec{\lambda}}$ admits deformations: in a particular degenerate situation, the enumerative problem breaks up into two parts which are identified with the ranks of $\mathbb{V}_{\mathfrak{s l}_{r+1}, \vec{\lambda}, \ell}$ and $\mathbb{V}_{\mathfrak{s l}_{\ell+1}, \vec{\lambda}^{T}, r}$, and Proposition 1.6(a) follows.

We first define a number of enumerative problems. We set $N=r+\ell+2$ and introduce the notation $I^{p}=\left\{i_{1}^{p}<\cdots<i_{r+1}^{p}\right\}$ for $p \in S=\left\{p_{1}, \ldots, p_{n}\right\}$, by the formula $\lambda_{i}^{(a)}=(\ell+1)+a-i_{a}^{p_{i}}$ for $a \in[r+1], i \in[n]$. Since $\vec{\lambda}$ is an $n$-tuple of normalized weights, $i_{r+1}^{p}=N$ and $i_{1}^{p} \neq 1$. Next, we will establish three enumerative interpretations, for $\operatorname{rk} \mathbb{A}_{\mathfrak{s l}_{r+1}, \vec{\lambda}}, \operatorname{rk} \mathbb{V}_{\mathfrak{s l}_{r+1}, \vec{\lambda}, \ell}$, and $\operatorname{rk} \mathbb{V}_{\mathfrak{s l}_{\ell+1}, \vec{\lambda}^{T}, r}$.

\subsection{Enumerative problems}

9.1.1 The enumerative problem corresponding to $\operatorname{rk} \mathbb{A}_{\mathfrak{s l}_{r+1}, \vec{\lambda}}$. We first note that $\mathbb{A}_{\mathfrak{s l}_{r+1}, \vec{\lambda}}$ and $\mathbb{A}_{\mathfrak{s l}_{\ell+1}, \vec{\lambda}^{T}}$ have the same rank (by their enumerative interpretation and Grassmann duality; see Section 2.3) and that these ranks equal the following enumerative number. Let $\mathcal{W}=\mathcal{O}^{\oplus N}$. 


\section{VANISHING AND IDENTITIES OF CONFORMAL BLOCKS DIVISORS}

Choose a general point $\mathcal{E} \in \mathrm{Fl}_{S}(\mathcal{W})$. The enumerative problem is: Count subbundles $\mathcal{V}$ of degree zero and rank $r+1$ of $\mathcal{W}$ such that $\mathcal{V}_{p_{i}} \in \Omega_{\lambda_{i}}\left(E_{\bullet}^{p_{i}}\right) \subseteq \operatorname{Gr}\left(r+1, \mathcal{W}_{p_{i}}\right), i=1, \ldots, n$.

Note that this is the same problem as counting classical intersections of generic translates of Schubert varieties (corresponding to $\lambda_{i}$ ) in a Grassmannian $\operatorname{Gr}(r+1, N)$.

9.1.2 The enumerative problem corresponding to $\mathrm{rk} \mathbb{V}_{\mathfrak{s l}_{r+1}, \vec{\lambda}, \ell}$. Let $\mathcal{W}^{\prime}=\mathcal{O}(1) \oplus \mathcal{O}^{\oplus N-2}$. Choose a general point $\mathcal{E}^{\prime} \in \mathrm{Fl}_{S}\left(\mathcal{W}^{\prime}\right)$. Count subbundles $\mathcal{V}$ of degree zero and rank $r+1$ of $\mathcal{W}^{\prime}$ such that $\Omega_{\lambda_{i}}\left(E_{\bullet}^{\prime p_{i}}\right) \subseteq \operatorname{Gr}\left(r+1, \mathcal{W}_{p_{i}}^{\prime}\right), i=1, \ldots, n$.

9.1.3 The enumerative problem corresponding to $\mathrm{rk} \mathbb{V}_{\mathfrak{s l}_{\ell+1}, \vec{\lambda}^{T}, r}$. The enumerative problem corresponding to $\mathbb{V}_{\mathfrak{s l}_{\ell+1}, \vec{\lambda}^{T}, r}$ is counting subbundles of $\mathcal{W}^{\prime}$ of degree zero and rank $\ell+1$ of $\mathcal{W}^{\prime}=$ $\mathcal{O}(1) \oplus \mathcal{O}^{\oplus N-2}$ subject to incidence conditions at the points $p \in S$. We "Grassmann dualize" this problem by considering the dual of quotients $\mathcal{V}=\left(\mathcal{W}^{\prime} / \mathcal{T}\right)^{*} \subseteq\left(\mathcal{W}^{\prime}\right)^{*}$. The association of $\mathcal{T}$ with $\mathcal{V}$ is one-one and we may write down equivalent conditions on $\mathcal{V}$.

The resulting enumerative problem is the following: Let $\overline{\mathcal{W}}=\mathcal{O}(-1) \oplus \mathcal{O}^{\oplus N-2}$. Choose a general point $\overline{\mathcal{E}} \in \mathrm{Fl}_{S}(\overline{\mathcal{W}})$. Count subbundles $\mathcal{V}$ of degree -1 and rank $r$ of $\overline{\mathcal{W}}$ such that $\mathcal{V}_{p_{i}} \in \Omega_{\lambda_{i}}\left(\bar{E}_{\bullet}^{p_{i}}\right) \subseteq \operatorname{Gr}\left(r+1, \overline{\mathcal{W}}_{p_{i}}\right), i=1, \ldots, n$.

\subsection{Outline of the proof of Proposition 1.6(a)}

The main idea is to degenerate the enumerative problem corresponding to $\mathbb{A}_{\mathfrak{s l}_{r+1}, \vec{\lambda}}$ by replacing $\mathcal{O}^{\oplus N}$ with its simplest degeneration $\mathcal{O}(1) \oplus \mathcal{O}(-1) \oplus \mathcal{O}^{\oplus(N-2)}$. The quot scheme corresponding to $\mathcal{O}^{\oplus N}$ (actually a Grassmannian) degenerates (flatly) into a union of two smooth quot schemes intersecting transversally.

For every bundle $\mathcal{W}$ of rank $N$ and degree zero, we can pose an enumerative problem (to ensure conservation of numbers, we will work with versions of the enumerative problems where one is looking for points in projective varieties; see Section 12): Fix a general $\mathcal{E} \in \mathrm{Fl}_{S}(\mathcal{W})$ and "count" coherent subsheaves $\mathcal{V} \subseteq \mathcal{W}$ of degree zero and rank $r+1$ such that $\mathcal{V}_{p} \rightarrow \mathcal{W}_{p} / E_{i_{a}^{p}}^{p}$ has kernel of dimension at least $a$ for all $a \in[r+1]$ and $p \in S$. Note that for some $\mathcal{W}$, the problem above is not enumerative; that is, the solution scheme is not of the expected dimension (or non-reduced). But we will show that for $\mathcal{O}^{\oplus N}$ and $\mathcal{O}(1) \oplus \mathcal{O}(-1) \oplus \mathcal{O}^{\oplus(N-2)}$, this problem is enumerative.

When $\mathcal{W}=\mathcal{O}^{\oplus N}$, this is the counting problem of Section 9.1.1. Now replace $\mathcal{O}^{\oplus N}$ with $\mathcal{O}(1) \oplus \mathcal{O}(-1) \oplus \mathcal{O}^{\oplus(N-2)}$. The degenerate enumerative number breaks up into two parts and we identify these parts as ranks of conformal blocks. We show that the enumerative counts are conserved in Section 9.8, and hence obtain equality (1).

\subsection{Quot schemes and degenerations}

Let Quot be the quot scheme of degree zero and rank $r+1$ subsheaves of the bundle $\mathcal{W}=$ $\mathcal{O}(1) \oplus \mathcal{O}(-1) \oplus \mathcal{O}^{\oplus N-2}$. Note that the surjection $\mathcal{W} \rightarrow \mathcal{O}(-1)$ and the inclusion $\mathcal{O}(1) \rightarrow \mathcal{W}$ are canonical (up to scalars). Hence the corresponding kernel and quotients are canonical.

Consider the evenly split sheaves $\mathcal{W}^{\prime}=\mathcal{O}(1) \oplus \mathcal{O}^{\oplus N-2} \subseteq \mathcal{W}$ and $\overline{\mathcal{W}}=\mathcal{W} / \mathcal{O}(1)$. Let Quot 1 be the (smooth) quot scheme of degree 0 rank $r+1$ subsheaves of $\mathcal{W}^{\prime}$ and Quot $_{2}$ the (smooth) quot scheme of degree -1 and rank $r$ subsheaves of $\overline{\mathcal{W}}$.

LEMmA 9.1. (i) We have Quot $=$ Quot $_{1} \cup$ Quot $_{2}$, where Quot ${ }_{1}$ and Quot $_{2}$ are smooth sub- 


\section{Belkale, Gibney and Mukhopadhyay}

schemes and $C=$ Quot $_{1} \cap$ Quot $_{2}$ is a subscheme of smaller dimension.

(ii) The scheme Quot is smooth in the complement of Quot $_{1} \cap$ Quot $_{2}$.

(iii) Points of Quot $\backslash$ Quot $_{1} \cap$ Quot $_{2}$ correspond to subbundles of $\mathcal{W}$.

(iv) The dimensions of Quot 1 and Quot $_{2}$ equal $(r+1)(\ell+1)$.

\subsection{Proof of Lemma 9.1}

Consider a $\mathcal{V} \subseteq \mathcal{W}$ such that $\mathcal{Q}=\mathcal{W} / \mathcal{V}$ is a point of Quot. Clearly $\mathcal{V}$ cannot have factors of the type $\mathcal{O}(a), a \geqslant 2$, as these do not admit non-zero maps to $\mathcal{W}$. Any map $\mathcal{O}(1) \rightarrow \mathcal{W}$ has image inside the standard copy $\mathcal{O}(1) \subseteq \mathcal{W}$. Therefore there cannot be more than one $\mathcal{O}(1)$ in such a $\mathcal{V}$.

The maps Quot $_{j} \rightarrow$ Quot, $j=1,2$, are clear. Let $\mathcal{V} \subseteq \mathcal{W}$ correspond to a point on Quot. If the composite $\mathcal{V} \rightarrow \mathcal{W} \rightarrow \mathcal{O}(-1)$ is the zero map, then the point is in Quot $_{1}$. Otherwise, $\mathcal{V}$ has a factor of $\mathcal{O}(1)$ which maps isomorphically to the canonical copy of $\mathcal{O}(1)$ in $\mathcal{W}$. So $\mathcal{V}$ is determined by the sub-sheaf $\mathcal{V} / \mathcal{O}(1) \subseteq \overline{\mathcal{W}}$, that is, a point of Quot $_{2}$.

Points in $C$ correspond to subsheaves of $\mathcal{W}^{\prime} / \mathcal{O}(1)$ of degree -1 and rank $r$. It is easy to show that

$$
\operatorname{dim} \text { Quot }_{1}=\operatorname{dim} \text { Quot }_{2}=\operatorname{dim} C+1 .
$$

Let $A=$ Quot $_{1} \backslash C \subseteq$ Quot and $B=$ Quot $_{2} \backslash C$. At points of $A, \mathcal{V}$ is of the form $\mathcal{O}^{\oplus r+1}$. In this case, clearly $H^{1}\left(\mathbb{P}^{1}, \mathcal{V}^{*} \otimes \mathcal{W}\right)=0$. Long exact sequences in cohomology imply that $H^{1}\left(\mathbb{P}^{1}\right.$, $\left.\mathcal{V}^{*} \otimes \mathcal{W}\right)=0$ surjects onto $H^{1}\left(\mathbb{P}^{1}, \mathcal{V}^{*} \otimes \mathcal{W} / \mathcal{V}\right)$, and therefore the last group is zero. Therefore Quot is smooth at such points.

At points of $B, \mathcal{V}$ is isomorphic to $\mathcal{O}(1) \oplus \mathcal{O}(-1) \oplus \mathcal{O}^{r-1}$ and $\mathcal{S}=\mathcal{O}(1) \oplus \mathcal{O}^{\oplus N-2}$ surjects onto $\mathcal{W} / \mathcal{V}$. It is easy to see that $H^{1}\left(\mathbb{P}^{1}, \mathcal{V}^{*} \otimes \mathcal{S}\right)=0$; hence the required vanishing $H^{1}\left(\mathbb{P}^{1}\right.$, $\left.\mathcal{V}^{*} \otimes \mathcal{W} / \mathcal{V}\right)=0$ follows. Therefore Quot is smooth at such points.

\subsection{Degeneration of the Gromov-Witten numbers}

With $\mathcal{W}$ as above, consider a generic point $\mathcal{E} \in \operatorname{Fl}(\mathcal{W})$. For $p \in S$, the fiber $\mathcal{W}_{p}$ has a canonical quotient $\tau_{p}: \mathcal{W}_{p} \rightarrow L_{p}$ (corresponding to $\tau: \mathcal{W} \rightarrow \mathcal{O}(-1)$ ) and a canonical line $M_{p} \subseteq \mathcal{W}_{p}$ (corresponding to $\mathcal{O}(1) \subseteq \mathcal{W}$ ). In addition, $\tau_{p}\left(M_{p}\right)=0$. The genericity of the flags implies that $E_{1}^{p}$ surjects onto $L_{p}$ via $\tau_{p}$ and $E_{1}^{p} \cap M_{p}=\{0\}$. The induced flags $\mathcal{E}^{\prime} \in \mathrm{Fl}_{S}\left(\mathcal{W}^{\prime}\right)$ (note $\operatorname{ker} \tau=\mathcal{W}^{\prime}$ ) are therefore suitably general. Note that $E_{\bullet}^{\prime p}$ is the flag

$$
0 \subseteq E_{2}^{p} \cap \mathcal{W}_{p}^{\prime} \subseteq E_{3}^{p} \cap \mathcal{W}_{p}^{\prime} \subseteq \cdots \subseteq E_{N}^{p} \cap \mathcal{W}_{p}^{\prime}=\mathcal{W}_{p}^{\prime}
$$

Similarly, the induced flags $\overline{\mathcal{E}} \in \mathrm{Fl}_{S}(\overline{\mathcal{W}})$ (note $\overline{\mathcal{W}}=\mathcal{W} / \mathcal{O}(1)$ ) are suitably generic. Note that $\bar{E}^{p}$ is the flag (here $\gamma: \mathcal{W} \rightarrow \mathcal{W} / \mathcal{O}(1)$ )

$$
0 \subseteq \gamma\left(E_{1}^{p}\right) \subseteq \gamma\left(E_{2}^{p}\right) \subseteq \cdots \subseteq \gamma\left(E_{N-1}^{p}\right)=\overline{\mathcal{W}}_{p}
$$

We analyze the (degenerate) enumerative problem in Sections 9.6 and 9.7 below.

\subsection{The part of the intersection in Quot $_{1}$}

Take a coherent subsheaf $\mathcal{V} \subseteq \operatorname{ker} \tau=\mathcal{W}^{\prime}$ with the corresponding induced (generic) flags. This enumerative problem is the same as counting subsheaves of $\mathcal{W}^{\prime}$ such that $\mathcal{V}_{p} \rightarrow \mathcal{W}_{p} / E_{i_{a}^{p}}^{p}$ has kernel of dimension at least $a$ for all $a \in[r+1]$ and $p \in S$. So we need $\mathcal{V}_{p} \rightarrow \mathcal{W}_{p}^{\prime} / E_{i_{a}^{p}}^{p} \cap \mathcal{W}_{p}^{\prime}$ to have kernel of dimension at least $a$. But $E_{i_{a}^{p}}^{p} \cap \mathcal{W}_{p}^{\prime}$ has rank $i_{a}^{p}-1$. Let $J^{p}=\left\{i_{1}^{p}-1<\cdots<i_{N}^{p}-1\right\}$ and let $E_{\bullet}^{\prime p}$ be the induced flag on $\mathcal{W}_{p}^{\prime}$. The conditions on $\mathcal{V}$ are therefore that $\mathcal{V}_{p} \rightarrow \mathcal{W}_{p}^{\prime} / E_{j_{a}^{p}}^{\prime p}$ has 


\section{VANISHING AND IDENTITIES OF CONFORMAL BLOCKS DIVISORS}

kernel of rank at least $a$. This is just the compactified enumerative problem of subsheaves of the evenly split bundle $\mathcal{W}^{\prime}$ of degree zero and rank $r+1$ with Schubert conditions given by $\lambda_{i}$ at $p_{i}$. Note that we have

$$
N-(r+1)+a-i_{a}^{p}=(N-1)-(r+1)+a-\left(i_{a}^{p}-1\right)=\lambda_{i}^{(a)}, \quad p_{i}=p .
$$

This is the enumerative problem 9.1.2. Using Section 12, we conclude that the intersection in Quot $_{1}$ has $\mathrm{rk}_{\mathfrak{s l}_{r+1}, \vec{\lambda}, \ell}$ points and that the intersection lies entirely in the open part $A$ (see Lemma $12.2(\mathrm{c}))$.

\subsection{The part of the intersection in Quot $_{2}$}

Take a coherent subsheaf $\overline{\mathcal{V}} \subseteq \overline{\mathcal{W}}$ with the corresponding induced (generic) flags. This enumerative problem is the same as counting subsheaves (of the evenly-split bundle $\overline{\mathcal{W}}$ ) such that, setting $\mathcal{V}$ to be the inverse image of $\overline{\mathcal{V}}$ under the surjection $\mathcal{W} \rightarrow \overline{\mathcal{W}}, \mathcal{V}_{p} \rightarrow \mathcal{W}_{p} / E_{i_{a}^{p}}^{p}$ has kernel of dimension at least $a$.

Let $\bar{E}^{p}$ be the induced flag on $\overline{\mathcal{W}}_{p}$ and let $\bar{E}_{N-1}^{p}=\overline{\mathcal{W}}_{p}$. By an elementary calculation, the kernel of $\overline{\mathcal{V}}_{p} \rightarrow \overline{\mathcal{W}}_{p} / \bar{E}_{i_{a}^{p}}^{p}$ is isomorphic to the kernel of $\mathcal{V}_{p} \rightarrow \mathcal{W}_{p} / E_{i_{a}^{p}}^{p}$ for $a \leqslant r$. For this, write exact sequences

$$
0 \rightarrow \mathcal{O}(1)_{p} \rightarrow \mathcal{V}_{p} \rightarrow \overline{\mathcal{V}}_{p} \rightarrow 0 \quad \text { and } \quad 0 \rightarrow \mathcal{O}(1)_{p} \rightarrow \mathcal{W}_{p} / E_{i_{\ell}^{p}}^{p} \rightarrow \overline{\mathcal{W}}_{p} / \bar{E}_{i_{\ell}^{p}}^{p} \rightarrow 0
$$

Also note that $\bar{E}_{i_{a}^{p}}^{p}$ has rank $i_{a}^{p}$.

So we want to count subsheaves of $\overline{\mathcal{W}}$ of rank $r$ and degree -1 with Schubert conditions given by the data $\lambda_{i}$ at $p=p_{i}$. We have

$$
\begin{gathered}
(N-1)-r+\ell-i_{a}^{p}=N-(r+1)+\ell-i_{a}^{p}=\lambda_{i}^{(a)}, \quad a \leqslant r, \\
N-(r+1)+(r+1)-i_{r+1}^{p}=0 .
\end{gathered}
$$

Therefore the part of the enumerative problem in Quot $_{2}$ is the enumerative problem 9.1.3. It has rk $\mathbb{V}_{\mathfrak{s l}_{\ell+1}, \vec{\lambda}^{T}, r}$ points, and these points lie entirely in $B$ (again using Lemma 12.2 (c)).

\subsection{Proof of equality (1)}

Consider a family of vector bundles $\mathcal{W}$ over $\mathbb{P}^{1} \times T$, where $T$ is a smooth curve such that $\mathcal{W}_{t}$ is isomorphic to $\mathcal{O}^{\oplus n}$ for $t \neq t_{0}$ and isomorphic to $\mathcal{O}(1) \oplus \mathcal{O}(-1) \oplus \mathcal{O}^{\oplus(N-2)}$ for $t=t_{0}$. Let $\mathcal{E}_{t_{0}} \in$ $\mathrm{Fl}_{S}\left(\mathcal{W}_{t_{0}}\right)$ be generic and consider a family of $\mathcal{E}_{t} \in \mathrm{Fl}_{S}\left(\mathcal{W}_{t}\right)$ specializing to $\mathcal{E}_{t_{0}}$ and specializing at a fixed point $t_{1}$ to a general element of $\mathrm{Fl}_{S}\left(\mathcal{W}_{t_{1}}\right)$ with $t_{1} \neq t_{0}$.

We have a relative quot scheme (of quotients of degree zero and rank $\ell+1$ of $\mathcal{W}_{t}$ ) $\pi: \widetilde{\text { Quot }} \rightarrow T$

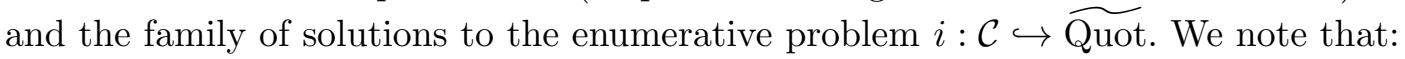

(i) The morphism $\pi$ is proper and $i$ is closed.

(ii) The morphism $\pi$ is smooth over $T-\left\{t_{0}\right\}$.

(iii) Each irreducible component of $\mathcal{C}$ has dimension at least one. This is because the map $\mathcal{C} \rightarrow T$ is the pull-back of an universal $\mathcal{C}^{\prime} \rightarrow T^{\prime}$ by a map $T \rightarrow T^{\prime}$. It turns out that $\mathcal{C}^{\prime}$ and $T^{\prime}$ have the same dimension and hence the assertion follows from standard properties of dimensions of fibers.

(iv) The morphism $\pi$ is smooth at $A \cup B \subseteq \pi^{-1}\left(t_{0}\right)$; it is not smooth at $C \subseteq \pi^{-1}\left(t_{0}\right)$.

(v) We have $(\pi \circ i)^{-1}\left(t_{0}\right) \subseteq A \cup B$ and $(\pi \circ i)^{-1}\left(t_{0}\right)$ is a reduced scheme consisting of finitely many points. 


\section{Belkale, Gibney and Mukhopadhyay}

By shrinking $T$ if necessary around $t_{0}$, we may assume that each component of $\mathcal{C}$ surjects onto $T$. Since $(\pi \circ i)^{-1}\left(t_{0}\right)$ is a reduced scheme consisting of finitely many points and each irreducible component of $\mathcal{C}$ has dimension at least one, we see that for $c \in \mathcal{C}$ over $t_{0}, \mathcal{O}_{C, c}$ is a discrete valuation ring (the Zariski tangent space has dimension at most one). By shrinking $T$ we may assume $\mathcal{C}$ to be smooth and equidimensional of dimension one. It is now easy to see that $\mathcal{C} \rightarrow T$ is finite and étale over a neighborhood of $t_{0}$. The generic fiber of $\mathcal{C} \rightarrow T$ has $\operatorname{rk}_{\mathfrak{s l}_{r+1}, \vec{\lambda}}$ points, and the special fiber over $t_{0}$ has $\mathrm{rk}_{\mathfrak{s l}_{\ell+1}, \vec{\lambda}^{T}, r}+\mathrm{rk} \mathbb{V}_{\mathfrak{s l}_{r+1}, \vec{\lambda}, \ell}$ points. Therefore equality (1) holds.

\subsection{Equality (1) stated in terms of quantum cohomology}

Assume that $\ell$ is the critical level for $\vec{\lambda}$. The rank $\operatorname{rk} \mathbb{A}_{\mathfrak{s l}_{r+1}, \vec{\lambda}}$ is the coefficient of the class of a point $[\mathrm{pt}]$ in the classical product

$$
\sigma_{\lambda_{1}} \cdot \sigma_{\lambda_{2}} \cdots \sigma_{\lambda_{n}} \in H^{*}(\operatorname{Gr}(r+1, r+\ell+2))
$$

Let $\lambda$ be the $(r+1) \times \ell$ Young diagram $(\ell, 0, \ldots, 0)$. Then $r k \mathbb{V}_{\mathfrak{s l}_{r+1}, \vec{\lambda}, \ell}$ (there is a similar expression for $\left.\mathrm{rk}_{\mathfrak{s l}_{\ell+1}, \vec{\lambda}^{T}, r}\right)$ is the coefficient of $q[\mathrm{pt}]$ in the small quantum product

$$
\sigma_{\lambda_{1}} \star \cdots \star \sigma_{\lambda_{n}} \star \sigma_{\lambda} \in Q H^{*}(\operatorname{Gr}(r+1, r+\ell+1)) .
$$

Note that the Grassmannian appearing in (11) is different from the one in (10). In terms of Gromov-Witten numbers (see Remark 3.6), the sum of

$$
\left\langle\sigma_{\lambda_{1}}, \ldots, \sigma_{\lambda_{n}}, \sigma_{(\ell, 0, \ldots, 0)}\right\rangle_{1} \quad \text { and } \quad\left\langle\sigma_{\lambda_{1}^{T}}, \ldots, \sigma_{\lambda_{n}^{T}}, \sigma_{(r, 0, \ldots, 0)}\right\rangle_{1}
$$

computed for $\operatorname{Gr}(r+1, r+\ell+1)$ and for $\operatorname{Gr}(\ell+1, r+\ell+1)$, respectively, equals the classical coefficient $\left\langle\sigma_{\lambda_{1}}, \ldots, \sigma_{\lambda_{n}}\right\rangle_{0}$ computed for $\operatorname{Gr}(r+1, r+\ell+2)$.

Remark 9.2. One may ask whether structure constants in the classical cohomology of a $G / P$ (the above is a special case where $G / P=\operatorname{Gr}(r+1, r+\ell+2)$ ) similarly decompose as sums of quantum cohomology structure constants (for possibly different groups). Any classical cohomology structure constant can be interpreted as the enumerative problem of counting suitable reductions of the structure group of the trivial principal $G$-bundle on $\mathbb{P}^{1}$ (subject to incidence conditions). We may replace the trivial principal $G$-bundle with its simplest degeneration and then look at the corresponding enumerative problem, and ask if it breaks up into smaller pieces.

\section{Conformal blocks as generalized theta functions}

\subsection{Notation}

A quasi-parabolic $\mathrm{SL}_{r+1}$-bundle on $\mathbb{P}^{1}$ is a triple $(\mathcal{V}, \mathcal{F}, \gamma)$ where $\mathcal{V}$ is a vector bundle on $\mathbb{P}^{1}$ of rank $r+1$ and degree zero with a given trivialization $\gamma: \operatorname{det} \mathcal{V} \stackrel{\sim}{\rightarrow} \mathcal{O}$, and $\mathcal{F}=\left(F_{\bullet}^{p_{1}}, \ldots, F_{\bullet}^{p_{n}}\right) \in \mathrm{Fl}_{S}(\mathcal{V})$ is a collection of complete flags on fibers over $p_{1}, \ldots, p_{n}$. Let $\mathcal{P} a r_{r+1}$ be the stack parameterizing quasi-parabolic $\mathrm{SL}_{r+1}$-vector bundles on $\mathbb{P}^{1}$.

\subsection{Generalized theta functions}

It is well known that conformal blocks for $\mathfrak{s l}_{r+1}$ on a smooth projective curve can be identified with the space of sections, called "generalized theta functions", of a suitable line bundle on the moduli space of vector bundles of rank $r+1$ with trivial determinant on that curve (see the survey [Sor96]). A parabolic generalization for $\mathfrak{s l}_{r+1}$ was proved in [Pau96]; we recall it now (but only for $\mathbb{P}^{1}$ ). 


\section{VANISHING AND IDENTITIES OF CONFORMAL BLOCKS DIVISORS}

Associated with the data $\vec{\lambda}=\left(\lambda_{1}, \ldots, \lambda_{n}\right) \in P_{\tilde{\ell}}\left(\mathfrak{s l}_{r+1}\right)$, we can form a line bundle $\mathcal{P}\left(\mathfrak{s l} l_{r+1}, \tilde{\ell}, \vec{\lambda}\right)$ on $\mathcal{P a r}_{r+1}$. The fiber over a point $(\mathcal{V}, \mathcal{F}, \gamma)$ is a tensor product

$$
D(\mathcal{V})^{\tilde{\ell}} \otimes \otimes_{i=1}^{n} \mathcal{L}_{\lambda_{i}}\left(\mathcal{V}_{p_{i}}, F_{\bullet}^{p_{i}}\right)
$$

where $D(\mathcal{V})$ is the determinant of cohomology of $\mathcal{V}$; that is, the line $\operatorname{det} H^{1}\left(\mathbb{P}^{1}, \mathcal{V}\right) \otimes \operatorname{det} H^{0}\left(\mathbb{P}^{1}, \mathcal{V}\right)^{*}$ and the lines $\mathcal{L}_{\lambda_{i}}\left(\mathcal{V}_{p_{i}}, F_{\bullet}^{p_{i}}\right)$ are as defined in Section 8.1.

It is known that the space of generalized theta functions is canonically identified (up to scalars) with the dual of the space of conformal blocks [Pau96]. Let $x=\left(\mathbb{P}^{1}, p_{1}, \ldots, p_{n}\right) \in \mathrm{M}_{0, n}$, then we have

$$
H^{0}\left(\mathcal{P a r}_{r+1}, \mathcal{P}\left(\mathfrak{s l}_{r+1}, \tilde{\ell}, \vec{\lambda}\right)\right) \stackrel{\sim}{\rightarrow}\left(\mathbb{V}_{\mathfrak{s l}_{r+1}, \vec{\lambda}, \tilde{\ell}}\right)_{x}^{*}
$$

The determinant of cohomology $D(\mathcal{V})$ of a vector bundle with a given trivialization $\gamma: \operatorname{det} \mathcal{V} \stackrel{\sim}{\rightarrow}$ carries a canonical element $\theta$. To construct $\theta$, let $P \in \mathbb{P}^{1}$ and consider

$$
0 \rightarrow \mathcal{V}(-P) \rightarrow \mathcal{V} \rightarrow \mathcal{V}_{P} \rightarrow 0,
$$

which sets up an isomorphism $D(\mathcal{V})=D(\mathcal{V}(-P))$ (note that det $\mathcal{V}_{P}$ has a given trivialization $\left.\gamma_{P}\right)$. But $\chi\left(\mathbb{P}^{1}, \mathcal{V}(-P)\right)=0$ and hence $D(\mathcal{V}(-P))$ carries a canonical theta section. Hence one gets an element $\theta$ of $D(\mathcal{V})$. This element $\theta$ does not depend upon $P$ because otherwise we would get non-trivial functions in $P \in \mathbb{P}^{1}$. We may also apply the isomorphism (12) at level one with vacuum representations at $p_{i}$ (that is, $\lambda_{i}=0$ ) to construct $\theta$.

The theta section of $D(\mathcal{V}(-P))$ vanishes along the locus where $\operatorname{dim} H^{0}(\mathcal{V}(-P)) \neq 0$. Since $\mathcal{V}$ is of degree zero, this is same as saying that $\theta$ vanishes at $\mathcal{V}$ if and only if $\mathcal{V}$ is non-trivial as a vector bundle.

Therefore one has a canonical section (also denoted by $\theta$ ) of the determinant of cohomology bundle on the moduli stack of vector bundles on $\mathbb{P}^{1}$ with trivialized determinant. Note that if $\mathcal{V}=\mathcal{O}^{\oplus r+1}$, then $\theta \in D(\mathcal{V})$ coincides with $1 \in \operatorname{det}\left(\mathbb{C}^{r+1}\right)^{*}=D(\mathcal{V})$.

Multiplication by $\theta \in D(\mathcal{V})$ sets up an injective map

$$
H^{0}\left(\mathcal{P a r}_{r+1}, \mathcal{P}\left(\mathfrak{s l}_{r+1}, \tilde{\ell}, \vec{\lambda}\right)\right) \rightarrow H^{0}\left(\mathcal{P a r}_{r+1}, \mathcal{P}\left(\mathfrak{s l}_{r+1}, \tilde{\ell}+1, \vec{\lambda}\right)\right) .
$$

Remark 10.1. The image of $(13)$ necessarily vanishes at points $(\mathcal{V}, \mathcal{F}, \gamma) \in \mathcal{P a r}_{r+1}$ with $\mathcal{V}$ not isomorphic to $\mathcal{O}^{\oplus r+1}$.

Note that $\mathcal{P} a r_{r+1}$ has a classical part, $\mathcal{P} a r_{r+1}^{c}$, the open substack where the underlying vector bundle is trivial. It is easy to see that

$$
H^{0}\left(\mathcal{P a r}_{r+1}^{c}, \mathcal{P}\left(\mathfrak{s l}_{r+1}, \tilde{\ell}, \vec{\lambda}\right)\right)=A_{r+1, \vec{\lambda}}^{*}
$$

via the map $\pi: \mathrm{Fl}(V)^{n} \rightarrow \mathcal{P} a r_{r+1}^{c}$, where $V$ is a vector space of dimension $r+1$ with trivialized determinant, which sets up $\mathcal{P} a r_{r+1}^{c}$ as a stack quotient $\operatorname{Fl}(V)^{n} / \operatorname{SL}(V)\left(\pi\right.$ pulls back $\mathcal{P}\left(\mathfrak{s l} l_{r+1}, \tilde{\ell}, \vec{\lambda}\right)$ to $\otimes_{i=1}^{n} \mathcal{L}_{\lambda_{i}}$. We therefore obtain injective maps

$$
H^{0}\left(\mathcal{P a r}_{r+1}, \mathcal{P}\left(\mathfrak{s l}_{r+1}, \tilde{\ell}, \vec{\lambda}\right)\right) \rightarrow A_{r+1, \vec{\lambda}}^{*},
$$

which are compatible with (13) (because the canonical section of $D(\mathcal{V})$ is 1 on $\operatorname{Fl}(V)^{n}$ with our normalization). Note that if the map (14) is an isomorphism for $\tilde{\ell}$, then it is also an isomorphism for $\tilde{\ell}+1$.

As a final compatibility (given (12)), we note that (14) is dual to the canonical surjection (up to scalars)

$$
\left.A_{r+1, \vec{\lambda}} \rightarrow\left(\mathbb{V}_{\mathfrak{s l}_{r+1}, \vec{\lambda}, \tilde{\ell}}\right)\right|_{x}
$$




\section{Belkale, Gibney and Mukhopadhyay}

\section{Proof of Theorem 1.8}

To prove that the composite

$$
\mathbb{V}_{\mathfrak{s l}_{r+1}, \vec{\lambda}, \ell}^{*} \rightarrow \mathbb{A}_{\mathfrak{s l}_{r+1}, \vec{\lambda}}^{*} \rightarrow \mathbb{A}_{\mathfrak{s l}_{\ell+1}, \vec{\lambda}^{T}} \rightarrow \mathbb{V}_{\mathfrak{s l}_{\ell+1}, \vec{\lambda}^{T}, r}
$$

is the zero map (see Section 8, Step 2), we use the enumerative interpretations of $\mathbb{A}_{\mathfrak{s} l_{r+1}, \vec{\lambda}}$ and $\mathbb{A}_{\mathfrak{s l}_{\ell+1}, \vec{\lambda}^{T}}$. In the setting of Sections 8.3 and 8.2, the duality maps are explicit for sections that are defined by the enumerative problem of classical Schubert calculus. To get explicit representatives for the images of $\mathbb{V}_{\mathfrak{s l}_{r+1}, \vec{\lambda}, \ell}$ in $\mathbb{A}_{\mathfrak{s l}_{r+1}, \vec{\lambda}}^{*}$, we use the (degenerate) enumerative problem of Section 9.5. We will show that there are natural isomorphisms (assuming that $\ell$ is the critical level for $\vec{\lambda}$ )

$$
\begin{aligned}
H^{0}\left(\mathcal{P a r}_{r+1}, \mathcal{P}\left(\mathfrak{s l}_{r+1}, \ell+1, \vec{\lambda}\right)\right) & \stackrel{\sim}{\rightarrow} A_{r+1, \vec{\lambda}}^{*}, \\
H^{0}\left(\mathcal{P a r}_{\ell+1}, \mathcal{P}\left(\mathfrak{s l}_{\ell+1}, r+1, \vec{\lambda}^{T}\right)\right) & \stackrel{\sim}{\rightarrow} A_{\ell+1, \vec{\lambda}^{T}}^{*} .
\end{aligned}
$$

By Section 3, we already know the equality of ranks in these isomorphisms. The resulting duality map

$$
H^{0}\left(\mathcal{P}_{a r+1}, \mathcal{P}\left(\mathfrak{s l}_{r+1}, \ell+1, \vec{\lambda}\right)\right) \stackrel{\sim}{\rightarrow} H^{0}\left(\mathcal{P} a r_{\ell+1}, \mathcal{P}\left(\mathfrak{s l}_{\ell+1}, r+1, \vec{\lambda}^{T}\right)\right)^{*}
$$

can be controlled in terms of a basis of sections coming from the enumerative problem from Section 9.5. This and Remark 10.1 allow us to chase elements through the composition (15).

Remark 11.1. The isomorphism (16) is an example of a "parabolic strange duality" isomorphism [Oud11]. It reads via (12), for $\sum\left|\lambda_{i}\right|=(r+1)(\ell+1)$, as

$$
\left.\left.\mathbb{V}_{\mathfrak{s l}_{r+1}, \vec{\lambda}, \ell+1}\right|_{x} ^{*} \stackrel{\sim}{\rightarrow} \mathbb{V}_{\mathfrak{s l}_{\ell+1}, \vec{\lambda}^{T}, r+1}\right|_{x}
$$

This isomorphism is identified with the isomorphism (5). It is important for our considerations to know that (5) is independent of any choices (of $x=\left(p_{1}, \ldots, p_{n}\right)$ ).

\subsection{Enlargement of the duality divisor D}

Let $\mathcal{M}=\operatorname{Par}_{r+1}, \mathcal{N}=\mathcal{P} r_{\ell+1}, \mathcal{M}^{c}=\mathcal{P a r}_{r+1}^{c}$, and $\mathcal{N}^{c}=\mathcal{P}^{c} r_{\ell+1}^{c}$. There are natural maps $\mathcal{M}^{c} \rightarrow \mathcal{M}$ and $\mathcal{N}^{c} \rightarrow \mathcal{N}$.

We will define a divisor $D \subseteq \mathcal{M} \times \mathcal{N}$ extending the divisor on $\mathcal{M}^{c} \times \mathcal{N}^{c}$ of Section 8.1. A point $(\mathcal{V}, \mathcal{F}, \mathcal{T}, \widetilde{\mathcal{G}})$ is in $D$ if and only if there is a non-zero map $\phi: \mathcal{V} \rightarrow \mathcal{Q}$ such that for $a \in[r+1]$ and $i \in[n]$,

$$
\phi\left(F_{a}^{p_{i}}\right) \subseteq G_{\ell+1-\lambda_{i}^{(a)}}^{p_{i}},
$$

where $\mathcal{Q}=\mathcal{T}^{*}$ and $\mathcal{G} \in \operatorname{Fl}(\mathcal{Q})$ are the flags induced from $\widetilde{\mathcal{G}} \in \operatorname{Fl}(\mathcal{T})$.

We can recast this into a "determinantal scheme". Define a locally free sheaf $\mathcal{K}$ on $\mathcal{M} \times \mathcal{N} \times \mathbb{P}^{1}$ as follows. The fiber of $\mathcal{K}$ at a point $b=\left(\mathcal{V}, \mathcal{F}, \mathcal{T}, \widetilde{\mathcal{G}}\right.$ ) is given by (as a bundle on $\mathbb{P}^{1}$ )

$$
0 \rightarrow \mathcal{K}_{b} \rightarrow \underline{\operatorname{Hom}}(\mathcal{V}, \mathcal{Q}) \rightarrow \oplus_{i=1}^{n} i_{p_{i}, *} \frac{\operatorname{Hom}\left(\mathcal{V}_{p_{i}}, \mathcal{Q}_{p_{i}}\right)}{\mathcal{P}_{\lambda_{i}}\left(F_{\bullet}^{p_{i}}, G_{\bullet}^{p_{i}}\right)} \rightarrow 0
$$

With the conditions that we have assumed, $\chi\left(\mathbb{P}^{1}, \mathcal{K}_{b}\right)=0$, so there is a canonical element $s \in D\left(\mathcal{K}_{b}\right)$ which vanishes if and only if $x \in D$. The determinant of cohomology $D\left(\mathcal{K}_{b}\right)$ is given by

$$
\otimes_{i=1}^{n}\left(\mathcal{L}_{\lambda_{i}}\left(F_{\bullet}^{p_{i}}\right) \otimes \mathcal{L}_{\lambda_{j}^{T}}\left(\widetilde{G}_{\bullet}^{p_{i}}\right)\right) \otimes D\left(\mathcal{V}^{*} \otimes \mathcal{T}^{*}\right)
$$




\section{VANISHING AND IDENTITIES OF CONFORMAL BLOCKS DIVISORS}

We can rewrite this as follows:

$$
\left(\otimes_{i=1}^{n}\left(\mathcal{L}_{\lambda_{i}}\left(F_{\bullet}^{p_{i}}\right)\right) \otimes D\left(\mathcal{V}^{*}\right)^{\ell+1}\right) \otimes\left(\otimes_{i=1}^{n} \mathcal{L}_{\lambda_{i}^{T}}\left(\widetilde{G}_{\bullet}^{p_{i}}\right) \otimes D\left(\mathcal{T}^{*}\right)^{r+1}\right)=\mathcal{A}_{\mathcal{V}, \mathcal{F}} \otimes \mathcal{B}_{\mathcal{T}, \widetilde{g}},
$$

where we have introduced the notation $\mathcal{A}=\mathcal{P}\left(\mathfrak{s l}_{r+1}, \ell+1, \vec{\lambda}\right)$ and $\mathcal{B}=\mathcal{P}\left(\mathfrak{s l}_{\ell+1}, r+1, \vec{\lambda}^{T}\right)$, used the canonical isomorphisms $D(\mathcal{V})=D\left(\mathcal{V}^{*}\right)$ and $D(\mathcal{T})=D\left(\mathcal{T}^{*}\right)$ (the determinants of $\mathcal{V}$ and $\mathcal{T}$ are trivialized), and the following lemma (see, for example, [Fal93], Theorem I.1).

Lemma 11.2. Let $\mathcal{V}, \mathcal{W}$ be vector bundles on $\mathbb{P}^{1}$. Suppose $\operatorname{deg} \mathcal{V}=\operatorname{deg} \mathcal{W}=0$. There is a natural isomorphism $D(\mathcal{V} \otimes \mathcal{W}) \rightarrow D(\mathcal{V})^{\mathrm{rk} \mathcal{W}} \otimes D(\mathcal{W})^{\mathrm{rk} \mathcal{V}}$ which specializes to the obvious one when $\mathcal{V}=\mathcal{O}^{\oplus \operatorname{rk} V}$ (note that $D(\mathcal{O})$ is canonically trivial).

Let $\mathcal{A}^{c}$ and $\mathcal{B}^{c}$ be the pull-backs of $\mathcal{A}$ and $\mathcal{B}$ under the maps $\mathcal{M}^{c} \rightarrow \mathcal{M}$ and $\mathcal{N}^{c} \rightarrow \mathcal{N}$, respectively. The duality divisor $D$ of $\mathcal{M} \times \mathcal{N}$ pulls back to the duality divisor of Section 8.1 on $\mathcal{M}^{c} \times \mathcal{N}^{c}$.

We introduce the following notation:

$$
\mathcal{A}_{\ell}=\mathcal{P}\left(\mathfrak{s l}_{r+1}, \ell, \vec{\lambda}\right), \quad \mathcal{A}_{r}=\mathcal{P}\left(\mathfrak{s l}_{\ell+1}, r, \vec{\lambda}^{T}\right) .
$$

To set the stage for the final verification, note that (comparing ranks and the enumerative interpretation of Section 9.1.1), the maps

$$
H^{0}(\mathcal{M}, \mathcal{A}) \stackrel{\sim}{\rightarrow} H^{0}\left(\mathcal{M}^{c}, \mathcal{A}^{c}\right) \stackrel{\sim}{\rightarrow} A_{r+1, \vec{\lambda}}^{*}
$$

are isomorphisms. There are also natural maps (see (13)) $H^{0}\left(\mathcal{M}, \mathcal{A}_{\ell}\right) \rightarrow H^{0}(\mathcal{M}, \mathcal{A})$. These maps are compatible with (18) (see (14)). Let us also note that each $y \in \mathcal{N}$ gives us an element "evaluation at $y$ " $\mathrm{ev}_{y} \in H^{0}(\mathcal{N}, \mathcal{B})^{*}$ which is well defined up to scalars.

Remark 11.3. By Remark 10.1, it follows that the image of ev $y$ vanishes in $H^{0}\left(\mathcal{N}, \mathcal{B}_{r}\right)^{*}$ if the underlying bundle of $y$ is non-trivial.

\subsection{Geometric data}

Recall the enumerative problem of Section 9.5 corresponding to a general point $\mathcal{E} \in \mathrm{Fl}_{S}(\mathcal{W})$ with $\mathcal{W}=\mathcal{O}(1) \oplus \mathcal{O}(-1) \oplus \mathcal{O}^{\oplus N-2}$. Let $\alpha=\operatorname{rk}_{\mathfrak{s l}_{r+1}, \vec{\lambda}, \ell}, \beta=\operatorname{rk} \mathbb{V}_{\mathfrak{s l}_{\ell+1}, \vec{\lambda}^{T}, r}$, so $\alpha+\beta=\operatorname{rk} \mathbb{A}_{\mathfrak{s l}_{r+1}, \vec{\lambda}}$. Solutions to the enumerative problem in $A \subseteq$ Quot produce vector bundles on $\mathbb{P}^{1}$ equipped with complete (induced) flags $\left(\mathcal{V}_{1}, \mathcal{F}_{1}\right), \ldots,\left(\mathcal{V}_{\alpha}, \mathcal{F}_{\alpha}\right)$, where $\mathcal{F}_{i} \in \mathrm{Fl}_{S}\left(\mathcal{V}_{i}\right)$. We obtain quotients equipped with complete (induced) flags $\left(\mathcal{Q}_{1}, \mathcal{G}_{1}\right), \ldots,\left(\mathcal{Q}_{\alpha}, \mathcal{F}_{\alpha}\right)$, where $\mathcal{Q}_{i}=\mathcal{W} / \mathcal{V}_{i}$ and $\mathcal{G}_{i} \in$ $\mathrm{Fl}_{S}\left(\mathcal{Q}_{i}\right)$. Note that the bundles $\mathcal{Q}_{i}$ are non-trivial since they have $\mathcal{O}(-1)$ as a direct summand (see Section 9.4).

Similarly, solutions to the enumerative problem in $B \subseteq$ Quot produce bundles and quotients (each equipped with flags at points of $S$ ): $\left(\mathcal{V}_{1}^{\prime}, \mathcal{F}^{\prime}\right), \ldots,\left(\mathcal{V}_{1}^{\prime}, \mathcal{F}_{\beta}^{\prime}\right)$ and $\left(\mathcal{Q}_{1}^{\prime}, \mathcal{G}_{1}\right), \ldots,\left(\mathcal{Q}_{\beta}^{\prime}, \mathcal{G}_{\beta}^{\prime}\right)$ (again $\mathcal{V}_{a}^{\prime} \subseteq \mathcal{W}$ and $\left.\mathcal{Q}_{a}^{\prime}=\mathcal{W} / \mathcal{V}_{a}^{\prime}\right)$. The bundles $\mathcal{V}_{a}$ are non-trivial since they have $\mathcal{O}(1)$ as a subsheaf (see Section 9.4).

Let $\left(\mathcal{T}_{i}, \widetilde{\mathcal{G}}_{i}\right)$ and $\left(\mathcal{T}_{a}^{\prime}, \widetilde{\mathcal{G}}_{a}\right)$ be the duals of $\left(\mathcal{Q}_{i}, \mathcal{G}_{i}\right)$ and $\left.\left(\mathcal{Q}_{a}, \mathcal{G}_{a}\right)\right)$, respectively, for $i \in[\alpha]$ and $a \in[\beta]$. The bundles $\mathcal{V}_{i}, \mathcal{T}_{i}, \mathcal{V}_{a}^{\prime}, \mathcal{T}_{a}^{\prime}, i \in[\alpha], a \in[\beta]$ are of degree zero. We choose and fix trivializations of their determinants. We therefore obtain

(i) points $x_{1}, \ldots, x_{\alpha} ; x_{1}^{\prime}, \ldots, x_{\beta}^{\prime} \in \mathcal{M}$, where $x_{i}$ and $x_{a}^{\prime}$ are the points $\left(\mathcal{V}_{i}, \mathcal{F}_{i}\right)$ and $\left(\mathcal{V}_{a}^{\prime}, \mathcal{F}_{a}^{\prime}\right)$, respectively;

(ii) points $y_{1}, \ldots, y_{\alpha} ; y_{1}^{\prime}, \ldots, y_{\beta}^{\prime} \in \mathcal{N}$, where $y_{i}$ and $y_{a}^{\prime}$ are the points $\left(\mathcal{T}_{i}, \widetilde{\mathcal{G}}_{i}\right)$ and $\left(\mathcal{T}_{a}^{\prime}, \widetilde{\mathcal{G}}_{a}^{\prime}\right)$, respectively. 


\section{Belkale, Gibney and Mukhopadhyay}

These points have the following properties:

(a) We have $s\left(x_{i}, y_{a}^{\prime}\right)=0$ and $s\left(x_{a}^{\prime}, y_{i}\right)=0$ for all $i \in[\alpha]$ and $a \in[\beta]$. This is because there are natural non-zero maps $\mathcal{V}_{i} \rightarrow \mathcal{Q}_{a}^{\prime}$ "inclusion in $\mathcal{W}$ followed by projection" (resp. $\mathcal{V}_{a}^{\prime} \rightarrow \mathcal{Q}_{j}$ ) which satisfy the conditions of (17) (with the corresponding flags $\mathcal{F}_{i}$ and $\mathcal{G}_{a}^{\prime}$ (resp. $\mathcal{F}_{a}^{\prime}$ and $\left.\mathcal{G}_{i}\right)$ ).

(b) We have $s\left(x_{a}^{\prime}, y_{b}^{\prime}\right) \neq 0$ if and only $a=b$, and $s\left(x_{i}, y_{j}\right) \neq 0$ if and only if $i=j, i, j \in[\alpha]$ and $a, b \in[\beta]$. This is a consequence of transversality in the enumerative problem (as in Remarks 8.2 and Lemma 12.3(b)).

(c) By Lemma 8.3, $\delta_{i}=s\left(., y_{i}\right)$ and $\gamma_{a}=s\left(., y_{a}^{\prime}\right), i \in[\alpha], a \in[\beta]$, form a basis for $H^{0}(\mathcal{M}, \mathcal{A})$.

The desired vanishing of $\mathbb{V}_{\mathfrak{s l}_{r+1}, \vec{\lambda}, \ell} \rightarrow \mathbb{V}_{\mathfrak{s l}_{\ell+1}, \vec{\lambda}^{T}, r}$ comes follows from the proposition below.

Proposition 11.4. The composition of the following maps is zero:

$$
H^{0}\left(\mathcal{M}, \mathcal{A}_{\ell}\right) \rightarrow H^{0}(\mathcal{M}, \mathcal{A}) \rightarrow H^{0}(\mathcal{N}, \mathcal{B})^{*} \rightarrow H^{0}\left(\mathcal{N}, \mathcal{B}_{r}\right)^{*}
$$

Proof. The image of a section $\tau \in H^{0}\left(\mathcal{M}, \mathcal{A}_{\ell}\right)$ in $H^{0}(\mathcal{M}, \mathcal{A})$ can be expressed as a linear combination of basis elements $\delta_{i}$ and $\gamma_{a}$. Now evaluate such an expression at the points $x_{a}^{\prime}$. By Remark 10.1, $\tau\left(x_{a}^{\prime}\right)=0$ because the vector bundle $\mathcal{V}_{a}$ underlying $x_{a}^{\prime}$ is non-trivial. Thus $\tau$ is a linear combination of the sections $\delta_{i}$ by properties (a) and (b). By Lemma 8.3, the duality map $H^{0}(\mathcal{M}, \mathcal{A}) \rightarrow H^{0}(\mathcal{N}, \mathcal{B})^{*}$ sends $\delta_{i}$ to ev $y_{i}$. Remark 11.3 gives that the image of ev $y_{i}$ in $H^{0}\left(\mathcal{N}, \mathcal{B}_{r}\right)^{*}$ is zero (because the vector bundle $\mathcal{Q}_{i}^{*}$ underlying $y_{i}$ is non-trivial). Hence the image of $\tau$ in $H^{0}\left(\mathcal{N}, \mathcal{B}_{r}\right)^{*}$ is zero, as desired.

Remark 11.5. The points $x_{a}^{\prime}$ in $\mathcal{M}$ have non-trivial underlying bundles, and are yet parabolicsemistable for the linearization $\mathcal{P}\left(\mathfrak{s l}_{r+1}, \ell+1, \vec{\lambda}\right)$. This is because of the existence of parabolic bundles $y_{a}^{\prime}$ and the non-existence of non-zero maps of parabolic bundles from $x_{a}^{\prime}$ to $y_{a}^{\prime}$ (using (b) above and a parabolic generalization of a method of Faltings; see [Bel08b, A.1]).

Remark 11.6. - The proof of the vanishing statement, Proposition 1.3, even for $\mathfrak{s l}_{2}$ (noted in [Fak12]) uses some representation theory of $\mathfrak{s l}_{2}$, and does not use explicit formulas [Sor96]. We are not able to even see, using formulas, that for $\ell$ large enough and fixed $\vec{\lambda}$, $c_{1}\left(\mathbb{V}_{\mathfrak{s l}_{r+1}, \vec{\lambda}, \ell}\right)=0$.

- Formula (a) in Proposition 1.6 also does not seem to follow from explicit rank formulas [Sor96] (even for $n=3$ ). For example, the "denominators" in the explicit formulas are $(r+1+\ell)$ on one side and $(r+1+\ell+1)$ on the other (in strange duality settings where such equalities follow from formulas, the denominators are the same).

- Assuming Proposition 1.3 and part (a) of Proposition 1.6, one could ask if part (b) follows from Fakhruddin's Chern class formulas. The factorization data required (in the formulas of [Fak12]) for $\mathbb{V}_{\mathfrak{s l}_{r+1}, \vec{\lambda}, \ell}$ and $\mathbb{V}_{\mathfrak{s l}_{\ell+1}, \vec{\lambda}^{T}, r}$ are not known to be related, even for $n=4$ (in particular, one cannot assume that the factorization data is again at critical level), so it is not clear how to proceed with a formulaic approach.

\section{Compactifications and Gromov-Witten numbers}

In Section 9.2, we consider intersections in the entire Quot scheme instead of an open subset as done in Gromov-Witten theory. The goal of this section is to show that compactifying parameter spaces does not change the enumerative numbers. The main result of this section is Proposition 12.4, a generalization of a result of Bertram [Ber97]. 


\section{VANISHING AND IDENTITIES OF CONFORMAL BLOCKS DIVISORS}

Let $\mathcal{W}$ be a vector bundle on $\mathbb{P}^{1}$ of rank $N$ and degree $-D$. Let $d$ and $\tilde{r}$ be integers with $N>\tilde{r}>0$ (and $d, D$ possibly negative). Define $\operatorname{Gr}(d, \tilde{r}, \mathcal{W})$ to be the moduli space of subbundles of $\mathcal{W}$ of degree $-d$ and rank $\tilde{r}$. This is an open subset of the quot scheme $\operatorname{Quot}(d, \tilde{r}, \mathcal{W})$ of quotients of $\mathcal{W}$ of degree $d-D$ and rank $N-\tilde{r}$.

Proposition 12.1. Suppose that $\mathcal{W}$ is evenly-split.

(i) The scheme $\operatorname{Quot}(d, \tilde{r}, \mathcal{W})$ is a smooth projective variety (possibly empty) of dimension $\tilde{r}(N-\tilde{r})+d N-D \tilde{r}$.

(ii) The open subset $\operatorname{Gr}(d, \tilde{r}, \mathcal{W}) \subseteq \operatorname{Quot}(d, \tilde{r}, \mathcal{W})$ is dense and connected.

(iii) The subset $\operatorname{Gr}^{o}(d, \tilde{r}, \mathcal{W})$ of $\operatorname{Gr}(d, \tilde{r}, \mathcal{W})$ consisting of evenly split subbundles $\mathcal{V} \subseteq \mathcal{W}$ such that $\mathcal{W} / \mathcal{V}$ is also evenly split is open and dense in $\operatorname{Gr}(d, \tilde{r}, \mathcal{W})$.

See Section 12.1 of [Bel08a] for a proof of the above (which is certainly well-known).

Consider a five-tuple $(d, \tilde{r}, D, N, I)$ where $d, D, \tilde{r}, N$ are as above and $I$ assigns to each $p \in S$ an element $I^{p} \in\left(\begin{array}{c}{[N]} \\ \tilde{r}\end{array}\right)$. We will use the notation $I^{p}=\left\{i_{1}^{p}<\cdots<i_{\tilde{r}}^{p}\right\}$ for $p \in \mathrm{S}$, and introduce Young diagrams $\lambda_{p}$ by the formula $\lambda_{p}^{(a)}=N-\tilde{r}+a-i_{a}^{p}$ for $a=1, \ldots, \tilde{r}$. Assume that the numerical condition (which leads to enumerative problems with finitely many solutions)

$$
\tilde{r}(N-\tilde{r})-D \tilde{r}+d N=\sum_{p \in S}\left|\lambda_{p}\right|
$$

holds. Let $\mathcal{W}$ be a vector bundle of degree $-D$ and $\operatorname{rank} N$. Let $\mathcal{E} \in \mathrm{Fl}_{S}(\mathcal{W})$. We fix the data of $\mathcal{E}$ and $\mathcal{W}$ in this section.

Form the natural morphism

$$
\pi: \operatorname{Gr}(d, \tilde{r}, \mathcal{W}) \rightarrow \prod_{i=1}^{n} \operatorname{Gr}\left(\tilde{r}, \mathcal{W}_{p_{i}}\right)
$$

and consider

$$
\Omega=\pi^{-1}\left(\prod_{i=1}^{n} \Omega_{I^{p_{i}}}\left(E_{\bullet}^{p_{i}}\right)\right) .
$$

Since $\prod_{i=1}^{n} \operatorname{Gr}\left(\tilde{r}, \mathcal{W}_{p_{i}}\right)$ is a homogeneous space, Kleiman transversality implies the following result.

Lemma 12.2. Suppose that $\mathcal{W}$ is evenly split and $\mathcal{E} \in \mathrm{Fl}_{S}(\mathcal{W})$ is general. Then:

(a) The set $\Omega$ is finite.

(b) The set $\Omega$ coincides with the intersection of inverse images of open Schubert cells $\pi^{-1}\left(\prod_{i=1}^{n} \Omega_{I^{p_{i}}}^{o}\left(E_{\bullet}^{p_{i}}\right)\right)$, and is therefore a transverse intersection.

(c) We have $\Omega \subseteq \operatorname{Gr}^{o}(d, \tilde{r}, \mathcal{W})\left(\operatorname{Gr}^{o}(d, \tilde{r}, \mathcal{W})\right.$ was defined in Proposition 12.1).

If $\mathcal{W}$ is not general, we have a weaker result by replacing $\operatorname{Gr}(d, \tilde{r}, \mathcal{W})$ with its smooth open (possibly empty) subset $\operatorname{Gr}^{s m}(d, \tilde{r}, \mathcal{W})$ of points where the tangent space is of the expected dimension (that is, $\mathcal{V} \subseteq \mathcal{W}$ such that $\operatorname{Ext}^{1}(\mathcal{V}, \mathcal{W} / \mathcal{V})=0$ ). The following lemma was used in Section 11.2.

Lemma 12.3. Suppose that $\mathcal{E} \in \mathrm{Fl}_{S}(\mathcal{W})$ is general.

(a) The intersection $\Omega \cap \mathrm{Gr}^{s m}(d, \tilde{r}, \mathcal{W})$ is a smooth and transverse intersection in $\mathrm{Gr}^{s m}(d, \tilde{r}, \mathcal{W})$ which coincides with $\mathrm{Gr}^{s m}(d, \tilde{r}, \mathcal{W}) \cap \pi^{-1}\left(\prod_{i=1}^{n} \Omega_{I^{p_{i}}}^{o}\left(E_{\bullet}^{p_{i}}\right)\right)$. 
(b) Suppose $\mathcal{V} \in \Omega \cap \operatorname{Gr}^{s m}(d, \tilde{r}, \mathcal{W})$. Let $\mathcal{Q}=\mathcal{W} / \mathcal{V}$, and let $\mathcal{F} \in \mathrm{Fl}_{S}(\mathcal{V}), \mathcal{G} \in \mathrm{Fl}_{S}(\mathcal{Q})$ be the induced flags. There are no non-zero maps $\phi: \mathcal{V} \rightarrow \mathcal{Q}$ such that for $a=1, \ldots, r+1$ and $i=1, \ldots, n$, the inclusion (17) holds.

Proof. Part (a) follows from Kleiman transversality by restricting the domain of $\pi$ to the smooth open subset $\operatorname{Gr}^{s m}(d, \tilde{r}, \mathcal{W})$. For part (b), note that the tangent space to $\operatorname{Gr}(d, \tilde{r}, \mathcal{W})$ at $\mathcal{V}$ is $\operatorname{Hom}(\mathcal{V}, \mathcal{Q})$, and the tangent spaces to Schubert cells are as described in Section 3. Now, part (b) follows from the transversality and zero-dimensionality of the intersection $\Omega$ at $\mathcal{V}$.

It is important to work with compact parameter spaces while degenerating enumerative problems. The set $\operatorname{Gr}(d, \tilde{r}, \mathcal{W})$ is not compact, but $\operatorname{Quot}(d, \tilde{r}, \mathcal{W})$ is a projective variety which contains $\operatorname{Gr}(d, \tilde{r}, \mathcal{W})$ as an open subset. We therefore consider the following modified enumerative problem:

- Let $\Omega^{\prime} \subseteq \operatorname{Quot}(d, \tilde{r}, \mathcal{W})$ be the set of subsheaves $\mathcal{V} \subseteq \mathcal{W}$ of degree $-d$ and rank $\tilde{r}$ such that for all $p \in S, a \in[\tilde{r}]$, the map $\mathcal{V}_{p} \rightarrow \mathcal{W}_{p} / E_{i_{a}^{p}}$ has kernel of dimension at least $a$.

The following is due to Bertram (Lemma 2.2A in [Ber97]) when $D=0$. We give an obvious extension of Bertram's proof for arbitrary $D$ in Section 12.1.

Proposition 12.4 [Ber97]. Suppose that $\mathcal{W}$ is evenly split and $\mathcal{E} \in \mathrm{Fl}_{S}(\mathcal{W})$ is general. Then $\Omega=\Omega^{\prime}$.

\subsection{Proof of Proposition 12.4}

We reproduce a variant of Bertram's proof, adapted to our notation. We stratify Quot $(d, \tilde{r}, \mathcal{W})$ with strata $\operatorname{Gr}(\epsilon)$ parameterized by functions $\epsilon: S \rightarrow \mathbb{Z}$ such that $0 \leqslant \epsilon(p) \leqslant \tilde{r}$. We define $\operatorname{Gr}(\epsilon)$ to be the (quot) scheme of subsheaves $\mathcal{V} \subseteq \mathcal{W}$ such that the map $\mathcal{V}_{p} \rightarrow \mathcal{W}_{p}$ has kernel $K_{p}$ of rank $\epsilon(p), p \in S$. Let $\mathcal{V}$ be a generic point in $\operatorname{Gr}(\epsilon) \cap \Omega^{\prime}$. Suppose that the subset $\mathcal{V}_{p} / K_{p}$ is a subspace of $\mathcal{W}_{p}$ in the Schubert cell of $\operatorname{Gr}\left(\tilde{r}-\epsilon(p), \mathcal{W}_{p}\right)$ corresponding to the $(\tilde{r}-\epsilon(p))$-tuple $J^{p}=\left\{j_{1}^{p}<\cdots<j_{\tilde{r}-\epsilon(p)}^{p}\right\}$. We clearly need $j_{a}^{p} \leqslant i_{a+\epsilon(p)}^{p}, a \in[\tilde{r}-\epsilon(p)]$.

One therefore gets that the codimension of the Schubert cell corresponding to $J^{p}$ is at least the codimension of the Schubert cell corresponding to $I^{p} \operatorname{minus} \sum_{a=1}^{\epsilon(p)}\left(N-\tilde{r}+a-i_{a}^{p}\right)$. The dimension of each irreducible component of $\operatorname{Gr}(\epsilon) \cap \Omega^{\prime}$ is therefore (by a simple calculation, using (19) and Kleiman transversality) at most

$$
\operatorname{dim} \operatorname{Gr}(\epsilon)-\operatorname{dim} \operatorname{Gr}(d, \tilde{r}, \mathcal{W})+\sum_{p \in S} \sum_{a=1}^{\epsilon(p)}\left(N-\tilde{r}+a-i_{a}^{p}\right) .
$$

Lemma 12.5. Suppose $\operatorname{Gr}(\epsilon) \neq \emptyset$. Then $\operatorname{Gr}(\epsilon)$ is smooth and connected, and

$$
\operatorname{dim} \operatorname{Gr}(\epsilon)=\operatorname{dim} \operatorname{Gr}(d, \tilde{r}, \mathcal{W})-N \sum_{p \in S} \epsilon(p)+\sum_{p \in S} \epsilon(p)(\tilde{r}-\epsilon(p)) .
$$

Given the lemma, we see that the quantity (21) is less than or equal to

$$
\sum_{p \in S} \sum_{a=1}^{\epsilon(p)}\left(a-i_{a}^{p}-\epsilon(p)\right)<0
$$

unless $\epsilon(p)=0$ for each $p$. Therefore the enumerative intersection takes place over the stratum corresponding to $\epsilon(p)=0$. This stratum has an open dense subset given by $\operatorname{Gr}(d, \tilde{r}, \mathcal{W})$. By Kleiman transversality, the intersection occurs entirely on this subset. 


\section{VANISHING AND IDENTITIES OF CONFORMAL BLOCKS DIVISORS}

Proof of Lemma 12.5. Consider the variety $\mathcal{A}$ of tuples $\left(K_{p_{1}}, \ldots, K_{p_{n}}, \mathcal{V}\right)$ where $\mathcal{V}$ is a coherent subsheaf of $\mathcal{W}$ of degree $-d$ and rank $r, n$ is the number of points in $S$, and $K_{p} \subset \mathcal{V}_{p}$ are $\epsilon(p)$ dimensional subspaces such that the composites $K_{p} \rightarrow \mathcal{V}_{p} \rightarrow \mathcal{W}_{p}$ are zero for each $p$. Our $\operatorname{Gr}(\epsilon)$ is an open subset of $\mathcal{A}$ and so it suffices to find the dimension of $\mathcal{A}$.

Given a datum $\left(K_{p_{1}}, \ldots, K_{p_{n}}, \mathcal{V}\right)$, we can define a coherent sheaf $\widetilde{\mathcal{V}}$ containing $\mathcal{V}$ as follows:

- The sheaf $\tilde{\mathcal{V}}$ coincides with $\mathcal{V}$ outside of $S$.

- In a neighborhood $U_{p}$ of $p \in S$, sections of $\widetilde{\mathcal{V}}$ are determined as follows. Let $t_{p}$ be a uniformizing parameter at $p$. Sections of $\widetilde{\mathcal{V}}$ in $U_{p}$ are meromorphic sections $s$ of $\mathcal{V}$ such that $t_{p} s$ is a regular section of $\mathcal{V}$ on $U$, with fiber at $p$ in $K_{p}$.

Clearly $\widetilde{\mathcal{V}}$ is a coherent subsheaf of $\mathcal{W}$. We also obtain subspaces $Q_{p}=\operatorname{im}\left(\mathcal{V}_{p} \rightarrow \widetilde{\mathcal{V}}_{p}\right) \subseteq \widetilde{\mathcal{V}}_{p}$ of rank $\tilde{r}-\epsilon(p)$ for all $p \in S$. Therefore one obtains a tuple $\left(Q_{p_{1}}, \ldots, Q_{p_{n}}, \widetilde{\mathcal{V}}\right)$. Let $\mathcal{B}$ be the scheme of such tuples. It is easy to see that $\left(K_{p_{1}}, \ldots, K_{p_{n}}, \mathcal{V}\right)$ can be recovered from $\left(Q_{p_{1}}, \ldots, Q_{p_{n}}, \widetilde{\mathcal{V}}\right.$ ) (and viceversa; see Lemma A.2 in [Bel08a]). The schemes $\mathcal{A}$ and $\mathcal{B}$ are therefore isomorphic. We calculate the dimension of $\mathcal{B}$. The dimension of the space of such $\widetilde{\mathcal{V}}$ is $\operatorname{dim} \operatorname{Gr}(d, r, \mathcal{W})-N \sum_{p \in S} \epsilon(p)$. Adding the dimensions of the Grassmann bundles of $Q_{p}$, we see that Lemma 12.5 holds.

\section{Involution identities}

There is an involution on $P_{\ell}(\mathfrak{g})$ which sends a weight $\lambda$ to the highest weight $\lambda^{*}=-w_{0}(\lambda)$ of $\left(V_{\lambda}\right)^{*}$, where $w_{0}$ is the longest element of the Weyl group of $\mathfrak{g}$. For example, if $\mathfrak{g}=\mathfrak{s l}_{r+1}$ is of type $A$, then $\lambda^{*}$ is given by $\lambda^{c}$ for any weight $\lambda$ represented by a Young diagram of size $(r+1) \times \ell$.

Proposition 13.1. On $\overline{\mathrm{M}}_{0, \mathrm{n}}$, we have $\mathbb{D}_{\mathfrak{g}, \vec{\lambda}, \ell}=\mathbb{D}_{\mathfrak{g}, \vec{\lambda}^{*}, \ell}$, where $\vec{\lambda} \in P_{\ell}(\mathfrak{g})^{n}$.

By results in [Fak12], Proposition 13.1 reduces to the case $n=4$.

Lemma 13.2. On $\overline{\mathrm{M}}_{0,4}$, we have $\operatorname{deg} \mathbb{V}_{\mathfrak{g}, \vec{\mu}, \ell}=\operatorname{deg} \mathbb{V}_{\mathfrak{g}, \overrightarrow{\mu^{*}, \ell}}$.

Proof (of Lemma 13.2). Let $\mu$ be any dominant integral weight of $\mathfrak{g}$ and let $\mu^{*}=-w_{0}(\mu)$. The Cartan Killing form is invariant under the action of the Weyl group of $\mathfrak{g}$, so $(\mu, \mu+2 \rho)=$ $\left(\mu^{*}, \mu^{*}+2 \rho\right)$, where $\rho$ is the half sum of positive roots. We conclude the proof using Corollary 3.5 in [Fak12], and the above.

The following lemma relates the critical and theta levels for $\mathfrak{s l}_{r+1}$. Here, $\vec{\lambda}$ is an $n$-tuple of normalized integral weights for $\mathfrak{s l}_{r+1}$ such that $r+1$ divides $\sum_{i=1}^{n}\left|\lambda_{i}\right|$. One also obtains a different proof, only for $\mathfrak{g}=\mathfrak{s l}_{r+1}$, of theta level vanishing (Remark 1.5); see (b) below.

LemMA 13.3. (a) $\theta\left(\mathfrak{s l}_{r+1}, \vec{\lambda}\right)=\frac{1}{2} \cdot\left(c\left(\mathfrak{s l}_{r+1}, \vec{\lambda}\right)+c\left(\mathfrak{s l}_{r+1}, \vec{\lambda}^{*}\right)\right)$.

(b) If $\ell>\theta\left(\mathfrak{s l}_{r+1}, \vec{\lambda}\right)$, then $\mathbb{D}_{\mathfrak{s l}_{r+1}, \vec{\lambda}, \ell}=c_{1}\left(\mathbb{V}_{\mathfrak{s l}_{r+1}, \vec{\lambda}, \ell}\right)=0$.

(c) If the weights $\lambda_{1}^{*}, \ldots, \lambda_{n}^{*}$ are a permutation of the weights $\lambda_{1}, \ldots, \lambda_{n}$, then $c\left(\mathfrak{s} \mathfrak{l}_{r+1}, \vec{\lambda}\right)=$ $\theta\left(\mathfrak{s l}_{r+1}, \vec{\lambda}\right)$.

Proof. To prove part (a), normalize $\lambda_{i}^{*}$ to obtain weights $\mu_{i}$. It is easy to see that

$$
\left|\mu_{i}\right|=(r+1) \ell-\left|\lambda_{i}\right|-\left(\ell-\lambda_{i}^{(1)}\right)(r+1)=(r+1) \lambda_{i}^{(1)}-\left|\lambda_{i}\right|,
$$

which yields part (a). It is easy to see that part (b) follows from part (a), and Propositions 13.1 and 1.3 (applied to $\vec{\lambda}$ and $\vec{\lambda}^{*}$ ). Finally, part (c) follows from part (a). 


\section{Belkale, Gibney and Mukhopadhyay}

\section{Strange duality and critical level symmetries}

Several rank-level (or "strange") dualities have been proposed by many authors (inspired by work in mathematical physics, for example [NT92, Bel08b, MO07, Oud11]), over smooth pointed curves. In genus zero these take the form of isomorphisms (well-defined up to scalars) over $\mathrm{M}_{0, n}$ of the form

$$
\left.\left.\mathbb{V}_{\mathfrak{g}_{1}, \vec{\lambda}, \ell_{1}}\right|_{x} \cong \mathbb{V}_{\mathfrak{g}_{2}, \vec{\mu}, \ell_{2}}\right|_{x} ^{*}
$$

for suitable conformal embeddings $\mathfrak{g}_{1} \oplus \mathfrak{g}_{2} \subseteq \mathfrak{g}$ and $x=\left(\mathbb{P}^{1}, p_{1}, \ldots, p_{n}\right) \in \mathrm{M}_{0, n}$. These projective isomorphisms are defined only on the interior $\mathrm{M}_{0, n}$. For example, there is an isomorphism on $\mathrm{M}_{0, n}$ [NT92, Oud11], well-defined up to scalars, of the form

$$
\left.\left.\mathbb{V}_{\mathfrak{s l}_{r+1}, \vec{\lambda}, \ell}\right|_{x} \cong \mathbb{V}_{\mathfrak{s l}_{\ell}, \vec{\mu}, r+1}\right|_{x} ^{*}
$$

( $\vec{\lambda} \leftrightarrow \vec{\mu}$ is essentially the transpose up to diagram automorphisms).

While our critical level symmetries are reminiscent of this "strange duality", the bundles $\mathbb{V}_{\mathfrak{s l}_{r+1}, \vec{\lambda}, \ell}$ and $\mathbb{V}_{\mathfrak{s l}_{\ell+1}, \vec{\lambda}^{T}, r}$ on $\overline{\mathrm{M}}_{0, \mathrm{n}}$ are not dual, and often have different ranks. Because they are both globally generated, their first Chern classes are base-point free, and hence effective. For two effective divisors to be dual would mean they are trivial.

Moreover, the classical duality (5) can (eventually) be viewed as a special case of the general strange duality (23) given above (see Remark 11.1). We could say that the critical level identities of this paper are orthogonality relations "via" the strange duality (23). So whenever both sides of (22) are classical, that is, coincide with co-invariants, there is perhaps a symmetry of conformal blocks divisors (at levels $\ell_{1}-1, \ell_{2}-1$ for $\mathfrak{g}_{1}$ and $\mathfrak{g}_{2}$ ).

However, in [Muk13], the third author studies identities that do come from standard level-rank dualities associated with conformal embeddings of affine Lie algebras. Very roughly speaking, a conformal embedding of affine Lie algebras $\mathfrak{g}_{1} \oplus \mathfrak{g}_{2} \subseteq \mathfrak{g}$ producing a level rank duality gives rise to a corresponding relation of conformal blocks divisors which takes the shape

$$
\mathbb{D}_{\mathfrak{g}_{1}, \vec{\lambda}, \ell_{1}}+\mathbb{D}_{\mathfrak{g}_{2}, \vec{\mu}, \ell_{2}}=c \cdot \mathbb{D}_{\mathfrak{g}, \vec{\nu}, 1}+E,
$$

where $E$ is a sum of boundary and $\psi$ classes and $c \neq 0$, determined by the embedding.

\section{ACKNOWLEDGEMENTS}

We would like to thank N. Fakhruddin, D. Jensen, L. Mihalcea, H-B. Moon, and C. Sherman for useful discussions. We are grateful to the referees for their comments and suggestions. Proposition 1.3 and statement (b) of Proposition 1.6 are a generalization and sharpening of conjectures made by the second author, and formed the starting point for this work.

\section{REFERENCES}

Bea96 A. Beauville, Conformal blocks, fusion rules and the Verlinde formula, in Proceedings of the Hirzebruch 65 Conference on Algebraic Geometry, Ramat Gan, 1993, Israel Math. Conf. Proc. vol. 9, 75-96, Bar-Ilan Univ., Ramat Gan, 1996.

Bel04 P. Belkale, Invariant theory of $\mathrm{GL}(n)$ and intersection theory of Grassmannians, Int. Math. Res. Not. 69 (2004), 3709-3721. http://dx.doi.org/10.1155/S107379280414155X.

Bel08a P. Belkale, Quantum generalization of the Horn conjecture, J. Amer. Math. Soc. 21 (2008), no. 2, 365-408. http://dx.doi.org/10.1090/S0894-0347-07-00584-X. 


\section{VANISHING AND IDENTITIES OF CONFORMAL BLOCKS DIVISORS}

Bel08b P. Belkale, The strange duality conjecture for generic curves, J. Amer. Math. Soc. 21 (2008), no. 1, 235-258 (electronic). http://dx.doi.org/10.1090/S0894-0347-07-00569-3.

Bel10 P. Belkale, The tangent space to an enumerative problem, in Proceedings of the International Congress of Mathematicians, vol. II, 405-426, Hindustan Book Agency, New Delhi, 2010.

Ber97 A. Bertram, Quantum Schubert calculus, Adv. Math. 128 (1997), no. 2, 289-305. http://dx. doi.org/10.1006/aima.1997.1627.

BGM13 P. Belkale, A. Gibney, and S. Mukhopadhyay, Nonvanishing of conformal blocks divisors, version of August 2013, available at https://sites.google.com/site/angelagibney/.

BK10 P. Belkale and S. Kumar, Eigencone, saturation and Horn problems for symplectic and odd orthogonal groups, J. Algebraic Geom. 19 (2010), no. 2, 199-242. http://dx.doi.org/10.1090/ S1056-3911-09-00517-7.

CT13 A.-M. Castravet and J. Tevelev, $\overline{\mathrm{M}}_{0, n}$ is not a Mori Dream Space, arXiv:1311.7673

Fak12 N. Fakhruddin, Chern classes of conformal blocks, in Compact moduli spaces and vector bundles, Contemp. Math., vol. 564, 145-176, Amer. Math. Soc., Providence, RI, 2012. http://dx.doi. org/10.1090/conm/564/11148

Fal93 G. Faltings, Stable G-bundles and projective connections, J. Algebraic Geom. 2 (1993), no. 3, $507-568$.

FSV94 B. Feigin, V. Schechtman, and A. Varchenko, On algebraic equations satisfied by hypergeometric correlators in WZW models. I, Comm. Math. Phys. 163 (1994), no. 1, 173-184. http://projecteuclid.org/euclid.cmp/1104270384

FSV95 B. Feigin, V. Schechtman, and A. Varchenko, On algebraic equations satisfied by hypergeometric correlators in WZW models. II, Comm. Math. Phys. 170 (1995), no. 1, 219-247. http://projecteuclid.org/euclid.cmp/1104272957.

Ful00 W. Fulton, Eigenvalues, invariant factors, highest weights, and Schubert calculus, Bull. Amer. Math. Soc. (N.S.) 37 (2000), no. 3, 209-249 (electronic). http://dx.doi.org/10.1090/ S0273-0979-00-00865-X.

GK14 J. González and K. Karu, Some non-finitely generated Cox rings, arXiv:1407.6344.

GW86 D. Gepner and E. Witten, String theory on group manifolds, Nuclear Phys. B 278 (1986), no. 3, 493-549. http://dx.doi.org/10.1016/0550-3213(86)90051-9.

Has03 B. Hassett, Moduli spaces of weighted pointed stable curves, Adv. Math. 173 (2003), no. 2, 316-352. http://dx.doi.org/10.1016/S0001-8708(02)00058-0.

Kle74 S. L. Kleiman, The transversality of a general translate, Compositio Mathematica 28 (1974), no. 3, 287-297. http://eudml.org/doc/89215.

Kly98 A. A. Klyachko, Stable bundles, representation theory and Hermitian operators, Selecta Math. (N.S.) 4 (1998), no. 4, 419-445. http://dx.doi.org/10.1007/s000290050037.

KT99 A. Knutson and T. Tao, The honeycomb model of $\mathrm{GL}_{n}(\mathbf{C})$ tensor products. I. Proof of the saturation conjecture, J. Amer. Math. Soc. 12 (1999), no. 4, 1055-1090. http://dx.doi.org/ 10.1090/S0894-0347-99-00299-4.

Les47 L. Lesieur. Les problèmes d'intersection sur une variété de Grassmann, C. R. Acad. Sci. Paris 225 (1947), 916-917.

MO07 A. Marian and D. Oprea, The level-rank duality for non-abelian theta functions, Invent. Math. 168 (2007), no. 2, 225-247. http://dx.doi.org/10.1007/s00222-006-0032-z.

Muk13 S. Mukhopadhyay, Rank-Level duality and Conformal Block divisors, arXiv:1308.0854.

NT92 T. Nakanishi and A. Tsuchiya, Level-rank duality of WZW models in conformal field theory, Comm. Math. Phys. 144 (1992), no. 2, 351-372. http://projecteuclid.org/euclid.cmp/ 1104249321.

Oud11 R. Oudompheng, Rank-level duality for conformal blocks of the linear group, J. Algebraic Geom. 20 (2011), no. 3, 559-597. http://dx.doi.org/10.1090/S1056-3911-2010-00529-2. 


\section{Belkale, Gibney and Mukhopadhyay}

Pau96 C. Pauly, Espaces de modules de fibrés paraboliques et blocs conformes, Duke Math. J. 84 (1996), no. 1, 217-235. http://dx.doi.org/10.1215/S0012-7094-96-08408-2.

Sor96 C. Sorger, La formule de Verlinde, Astérisque 237, Exp. No. 794, 3, 87-114, 1996. Séminaire Bourbaki, Vol. 1994/95.

Swi10 D. Swinarski, conformalblocks: a macaulay2 package for computing conformal block divisors, 2010, Version 1.1, available at http://www . math.uiuc.edu/Macaulay2/.

Tsu93 Y. Tsuchimoto, On the coordinate-free description of conformal blocks, J. Math. Kyoto. Univ. 1 (1993), 29-49.

TUY89 A. Tsuchiya, K. Ueno, and Y. Yamada, Conformal field theory on universal family of stable curves with gauge symmetries, in Integrable systems in quantum field theory and statistical mechanics, Adv. Stud. Pure Math., vol. 19, 459-566, Academic Press, Boston, MA, 1989.

Wit95 E. Witten, The Verlinde algebra and the cohomology of the Grassmannian, in Geometry, topology, ${ }^{3}$ physics, Conf. Proc. Lecture Notes Geom. Topology, IV, 357-422, Int. Press, Cambridge, MA, 1995.

Prakash Belkale belkale@email.unc.edu

Department of Mathematics, University of North Carolina, Chapel Hill, NC 27599, USA

Angela Gibney agibney@math.uga.edu

Department of Mathematics, University of Georgia, Athens, GA 30602, USA

Swarnava Mukhopadhyay swarnava@umd.edu

Department of Mathematics, University of Maryland, College Park, MD 20742, USA 